\title{
Spatial phase differences can drive apparent motion
}

\author{
ALLISON B. SEKULER and PATRICK J. BENNETT \\ University of Toronto, Toronto, Ontario, Canada
}

\begin{abstract}
Can shape differences drive apparent motion? Results from previous research are equivocal. Much of the confusion may be due to the use of relatively complex stimuli: letters or geometric shapes, comprising many spatial frequencies, phases, orientations, and contrasts. We focus on relatively simple stimuli: Gaussian damped $f+n f$ compound sinewave gratings. We examine whether relative phase differences, which are critical for shape perception, can drive apparent motion. We find that some, but not all, phase differences can drive apparent motion. Specifically, stimuli that are easily discriminable and perceptually dissimilar can affect the solution of the correspondence problem. In this case, observers consistently perceive stimuli in one frame moving to the position of perceptually similar stimuli in the next frame. This general result holds over a wide range of spatial frequencies, orientations, and contrasts. Implications for theories of motion processing are discussed.
\end{abstract}

Do shape differences affect the perception of apparent motion? Results from previous experiments are equivocal. Some researchers have suggested that the perception of apparent motion can be affected by shape (Caelli, Manning, \& Finlay, 1993; He \& Nakayama, 1994; Koriat, 1994; Mack, Klein, Hill, \& Palumbo, 1989; Orlansky, 1940; Shechter, Hochstein, \& Hillman, 1988), but others have suggested that shape perception plays little, if any, role in apparent motion (Burt \& Sperling, 1981; Kolers, 1972; Navon, 1976; Prazdny, 1986; Ramachandran, Ginsburg, \& Anstis, 1983; Werkhoven, Sperling, \& Chubb, 1993). To some extent, the different conclusions may reflect differences in experimental methods. In addition, and perhaps more importantly, the previous studies often used complex stimuli: letters or geometric forms, comprising many spatial frequencies, relative phases, contrasts, and orientations.

Green (1986) suggested that a coherent explanation of all apparently conflicting results might be possible within the framework of the multiple-channel model of the human visual system. To this end, Green used a standard competitive motion paradigm to determine the extent to which simple stimulus differences in spatial frequency, orientation, or phase could "drive" apparent motion. In the present context, " $\mathrm{X}$ drives apparent motion" is short-

Portions of this paper were presented at the 1992 meeting of the Association for Research in Vision and Ophthalmology (Sekuler, Persaud, \& Bennett, 1992). This research was supported by a University of Toronto Grant and NSERC Grant OGP0105494 to A.B.S., and by NSERC Grant OGP0042133 to P.J.B. We thank Shin'ya Nishida, Myron Braunstein, and an anonymous reviewer for their invaluable comments on earlier versions of this manuscript, and Angela Persaud for her assistance in data collection. Correspondence should be addressed to A. B. Sekuler, Department of Psychology, University of Toronto, Toronto, ON, Canada M5S1A1 (e-mail: sekuler@psych. utoronto.ca). hand meaning: When stimuli differ on Dimension $X$, the difference between those stimuli can be used to solve the correspondence problem for apparent motion; specifically, from frame $n$ to frame $n+1$, Stimulus Al is perceived to move to the position of Stimulus A2, which most closely matches A1 in terms of Dimension X. Figure 1 shows a schematic example of the competitive motion paradigm used in Green's, and our own, experiments to determine the factors that can drive apparent motion. Two pairs of stimuli, represented by A and B, are presented in each frame. ${ }^{1}$ From one frame to the next, the stimulus configuration is rotated by $45^{\circ}$. Within this competitive motion paradigm, then, proximity cues cannot be used to solve the correspondence problem because the distances between a stimulus in frame $n$ and the two closest stimuli in frame $n+1$ are identical. If the differences between Stimuli A and B cannot be used to drive apparent motion, one would expect that subjects would be equally likely to perceive clockwise or counterclockwise motion. However, if the differences between Stimuli $A$ and $B$ can be used to drive apparent motion, one would expect that, for the situation illustrated here, subjects would consistently perceive counterclockwise motion.

Green's stimuli were sinusoidal gratings presented within a Gaussian envelope, and Stimuli A and B differed in terms of spatial frequency content, orientation, absolute phase, or relative phase. Absolute phase refers to the positioning of a stimulus with respect to the envelope, and relative phase refers to the relative positioning of different component gratings with respect to each other. Green found that differences in spatial frequency and orientation could drive apparent motion, but differences in absolute and relative spatial phase could not. On the basis of his results, Green suggested that previous contradictory results could be reconciled by considering the relative spatial frequency and orientation content of different complex stimuli. 


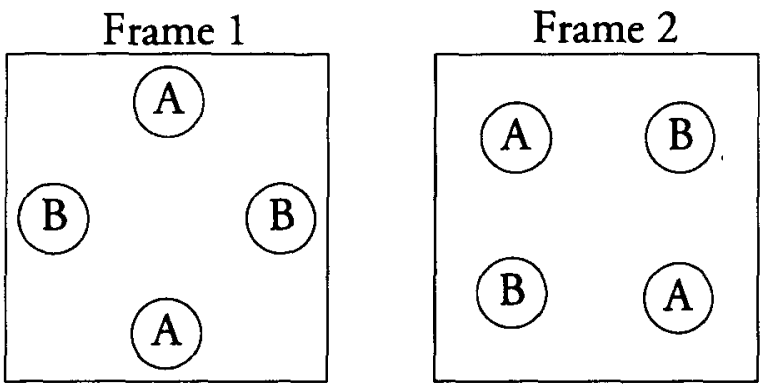

Figure 1. Schematic example of the motion competition paradigm we used. See text for details.

In a sense, Green's results support the notion that shape differences cannot drive apparent motion. Although spatial frequency and orientation clearly have roles in pattern detection and recognition, spatial phase appears to be the dominant cue in determining our perception of an object's shape (Piotrowski \& Campbell, 1982). Figure 2 illustrates how luminance profiles change with relative phase shifts. Each luminance profile in Figure 2 is the Gaussian modulated sum of two sinewave gratings with spatial frequencies $f$ and $2 f$. Although these stimuli vary along only one dimension, the luminance at any point in two-dimensional space (as for our stimuli) is described by

$$
\begin{aligned}
L(x, y)= & L_{\text {avg }}\left[1+G(x, y) C_{1} \cos \left(2 \pi f_{1} x-\emptyset_{a}\right)\right. \\
& \left.+G(x, y) C_{2} \cos \left(2 \pi f_{2} x-\left(f_{2} / f_{1}\right) \emptyset_{a}-\emptyset_{r}\right)\right],
\end{aligned}
$$

where $L_{\text {avg }}$ is the space-average luminance, $C_{1}$ and $C_{2}$ are the Michelson contrasts for $f_{1}$ and $f_{2}$, respectively, $\varnothing_{a}$ is the absolute phase of $f_{1}, \emptyset_{r}$ is the relative phase of $f_{2}$, and

$$
G(x, y)=\exp \left[-\left(x^{2}+y^{2}\right) / 2 s^{2}\right],
$$

where $s$ is the standard deviation of the Gaussian distribution.

In Figure $2, f_{1}$ is set to 0.5 cycles per degree (cpd), $f_{2}$ is $1.0 \mathrm{cpd}, C_{1}$ and $C_{2}$ are both 0.3 . The relative phase of $f_{2}, \varnothing_{r}$, is shifted by $-90^{\circ}(-\pi / 2)$ from one panel to the next. By varying the relative phase of $f_{2}$, the subject will perceive dark or light bars, left or right edges, ${ }^{2}$ or various intermediate percepts. Because relative phase differences influence our perception of shape, one would expect that if shape differences can drive apparent motion, then spatial phase differences in relatively simple grating stimuli should also drive apparent motion. Therefore, in the present experiments, we reexamined whether relative phase differences can drive apparent motion.

As Figure 2 suggests, not all equivalent phase shifts result in the same perceptual differences. The fundamental differences between Figures $2 \mathrm{~A}$ and $2 \mathrm{C}$ and between Figures 2B and 2D are both $180^{\circ}$ phase shifts of $f_{2}$. However, Figure 2A versus Figure $2 \mathrm{C}$ is a dark/light bar discrimination, while Figure $2 \mathrm{~B}$ versus Figure 2D is a left/right edge discrimination. In fact, these two $180^{\circ}$ phase differences are not equally discriminable: $0^{\circ}-180^{\circ}$ discriminations are easier to make than $90^{\circ}-270^{\circ}$ discriminations (Bennett \& Banks, 1987, 1991; Field \& Nachmias, 1984). ${ }^{3}$ In addition to this variation with base phase, the ability to discriminate phase differences also varies with orientation, spatial frequency, and eccentricity. The relative insensitivity to $90^{\circ}-270^{\circ}$ phase shifts can sometimes be reduced by orienting stimuli radially with respect to fixation or by decreasing eccentricity (Bennett \& Banks, 1991). Furthermore, all phase differences in $f+n f$ compound gratings become more difficult to discriminate as $n$ increases (Bennett, 1993); so, for example, $0^{\circ}-180^{\circ}$ phase differences may be more difficult to discriminate for $f+3 f$ than for $f+2 f$. In addition to discrimination differences, different $180^{\circ}$ phase shifts differ in their perceived similarity: $0^{\circ}-180^{\circ}$ phase differences are judged to be less similar to one another than $90^{\circ}-270^{\circ}$ phase differences (Kahana \& Bennett, 1994).

The fact that not all $180^{\circ}$ phase differences are equally discriminable or equally dissimilar is critical when considering the stimuli used in Green's (1986) experiments. The differences between the stimuli Green used were generally not the most perceptually salient. Therefore, Green's finding that phase differences could not drive apparent motion might be explained simply in terms of low discriminability or high similarity.

Green's stimuli were not oriented radially with respect to fixation, and he tested only $90^{\circ}-270^{\circ}$ phase differences for $f+2 f$ compound gratings, whereas he tested $0^{\circ}-180^{\circ}$ phase differences only for $f+3 f$ compound gratings. In addition, the stimuli were necessarily located in the near periphery, since Green was interested in the phenomenon of "long-range" apparent motion. Although Green presented no discriminability or perceptual similarity measures for his stimuli, the results of research on static phase discrimination suggest that Green's stimulus pairs would have been difficult to discriminate and perceived as highly similar. If this were the case, one would not necessarily expect that the physical difference between stimulus pairs could drive apparent motion. The critical question, and the question we begin to address in Experiment 1, is whether discriminable phase differences can drive apparent motion.

\section{EXPERIMENT 1}

As indicated above, the relative discriminability of $180^{\circ}$ relative phase shifts in $f+2 f$ compound stimuli depends on a number of factors, including the base phase of $2 f$ and stimulus orientation. The goal of Experiment 1 was to determine whether any phase differences could drive apparent motion. To this end, we chose a relatively low fundamental frequency $(0.5 \mathrm{cpd})$, so that the range of spatial frequencies examined would provide optimal sensitivity for the eccentricity tested (see Banks, Sekuler, \& Anderson, 1991; Rovamo, Virsu, \& Nasanen, 1978). We also varied the interstimulus interval (ISI) over a wide range (15-105 msec) because we did not know 

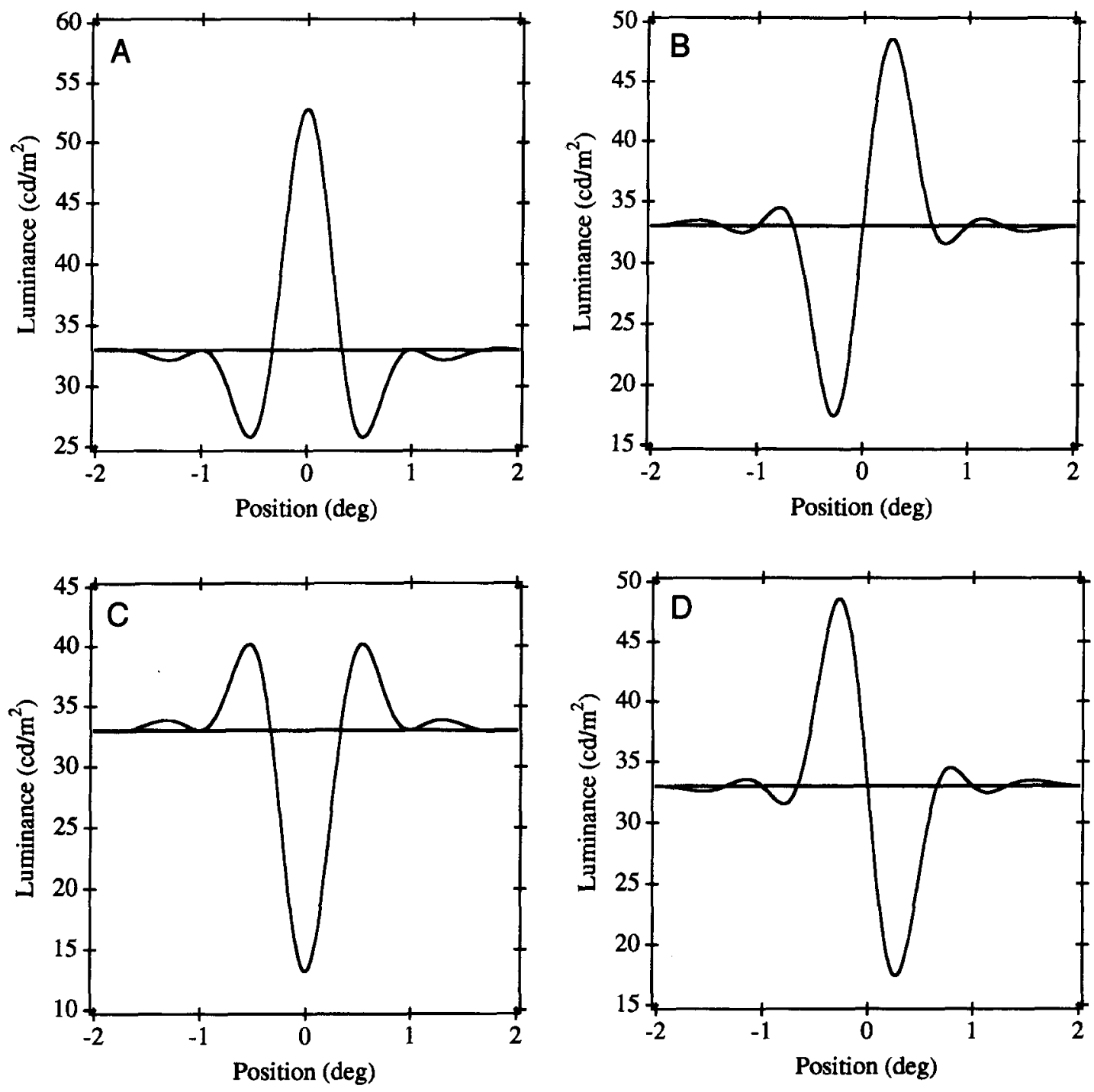

Figure 2. Gaussian damped luminance profiles for $f+2 f$ compound sinusoidal gratings with $f_{1}=0.5 \mathrm{cpd}, f_{2}=1.0 \mathrm{cpd}$, $C_{1}=C_{2}=0.3$, and $s=0.6$ (see Equations 1 and 2). Panel A: $\theta_{a}=0^{\circ}, \theta_{r}=0^{\circ}$. Panel B: $\theta_{a}=90^{\circ}, \theta_{r}=270^{\circ}$. Panel C: $\theta_{a}=180^{\circ}, \theta_{r}=180^{\circ}$; Panel D: $\theta_{a}=270^{\circ}, \theta_{r}=90^{\circ}$.

a priori what the optimal ISI would be, and we presented the stimuli in several different orientations.

\section{Method}

Subjects. Five subjects participated in this experiment, 3 of whom (S.K., S.M., and R.W.) were naive with respect to the purpose of the experiment. All subjects had normal or corrected-tonormal visual acuity. The naive subjects were paid for their participation.

Stimuli and Apparatus. Stimuli were generated by a Macintosh II with a $50-\mathrm{MHz}$ accelerator, a Macintosh IIci, or a Macintosh IIf and were presented on an Apple 13-in. high-resolution color monitor. The monitor's resolution was 72 pixels per inch ( 28.3 pixels per centimeter), and the frame rate was $67 \mathrm{~Hz}$, noninterlaced. Luminance was linearized with lookup tables, and the entire 8-bit range produced a maximum Michelson contrast of $95 \%$. Stimuli were Gaussian modulated compound sinusoids (Gabor patches) as defined in Equation 1; the horizontal and vertical standard devia- tions of the Gaussian were both set to $0.6, f\left(f_{1}\right)$ and $2 f\left(f_{2}\right)$ were set to 0.5 and $1.0 \mathrm{cpd}$, respectively, and both contrasts, $C_{f}$ and $C_{2 f}$, were set to $30 \%$. $L_{\text {avg }}$ was approximately $33 \mathrm{~cd} / \mathrm{m}^{2}$. Stimulus types $A$ and $B$ (see Figure 1) differed in the spatial phase of the second harmonic relative to the fundamental frequency. In the cosine phase condition, $\emptyset_{r}$ was either $0^{\circ}$ or $180^{\circ}$. In the sine phase condition, $\varnothing_{r}$ was $90^{\circ}$ or $270^{\circ}$. For all stimuli, absolute phase, $\varnothing_{a}$, was adjusted so that the peak of the pattern energy function ${ }^{4}$ (Morrone $\&$ Owens, 1987) was aligned with the center of the Gaussian envelope; thus, the stimuli were symmetric with respect to the envelope's center. Note that, for both cosine phase and sine phase conditions, we compared $180^{\circ}$ relative phase differences. Figure 2 shows the luminance profiles of the two cosine phase and two sine phase stimuli. The base phase for the two cosine stimuli was either $0^{\circ}$ or $180^{\circ}$ (Figures $2 \mathrm{~A}$ and $2 \mathrm{C}$, respectively), and that for the sine stimuli was either $270^{\circ}$ or $90^{\circ}$ (Figures $2 \mathrm{~B}$ and $2 \mathrm{D}$, respectively). The subjects viewed the stimuli binocularly from a distance of $57 \mathrm{~cm}$, with a head/chinrest stabilizing the head. From this viewing distance, the visible portion of each Gabor patch subtended ap- 
A

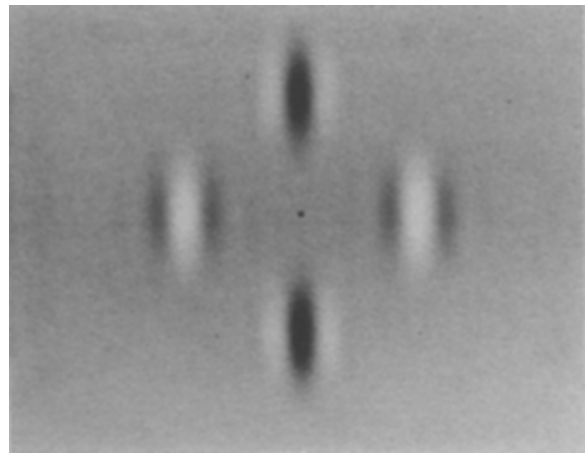

B

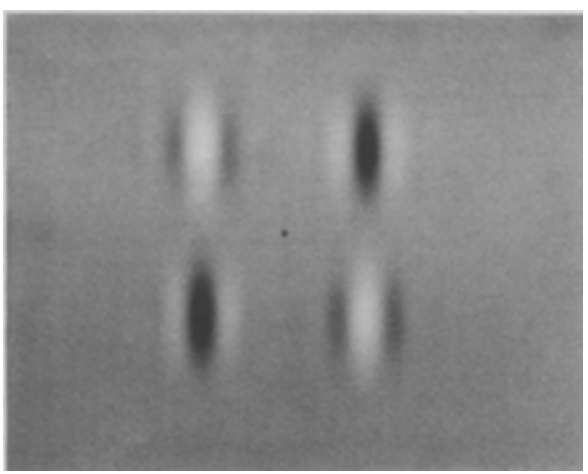

C

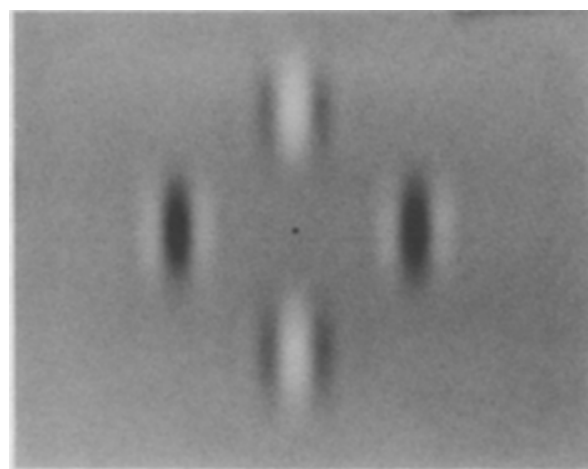

D

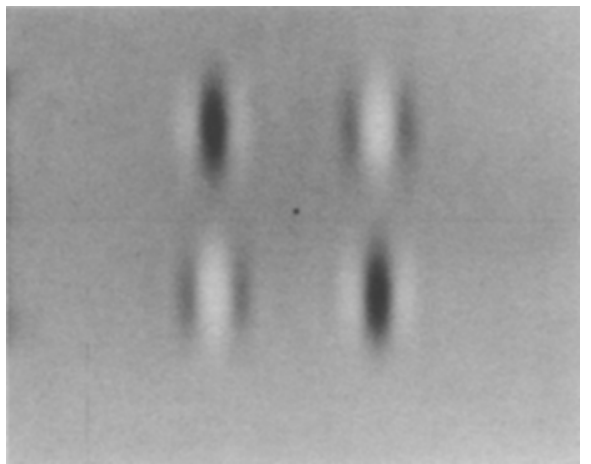

$E$

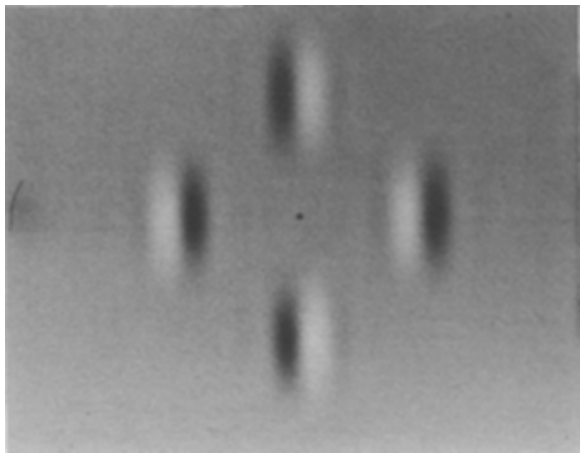

F

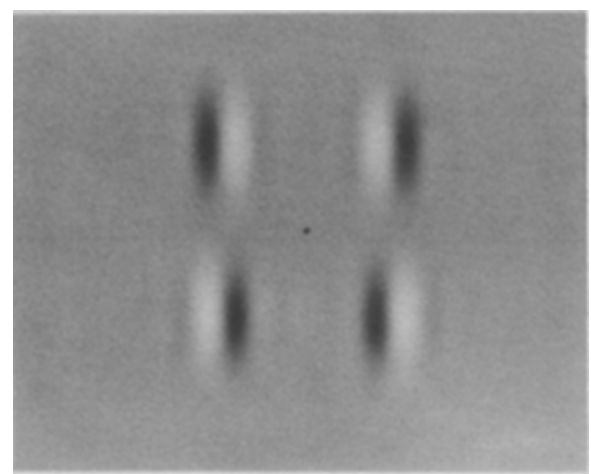

G

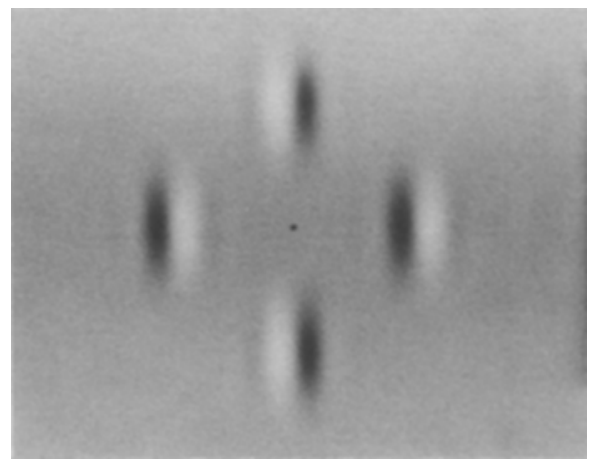

$\mathrm{H}$

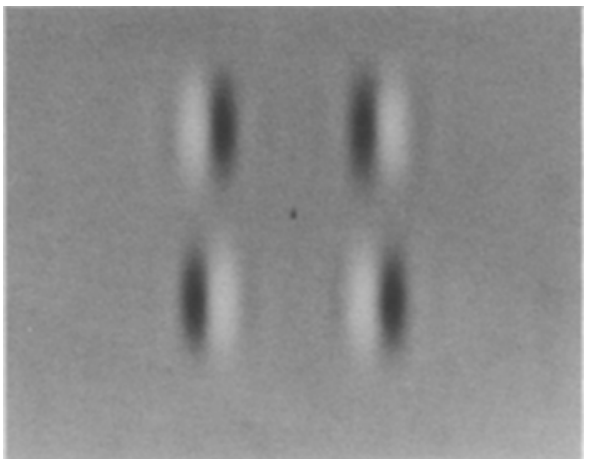

Figure 3. Photographs of four frames of the eight-frame sequence for each of the cosine phase (A-D) and sine phase $(\mathrm{E}-\mathrm{H})$ Ferris wheel conditions. "Correct" direction for the stimuli shown here is clockwise for cosine phase stimuli and counterclockwise for sine phase stimuli. 


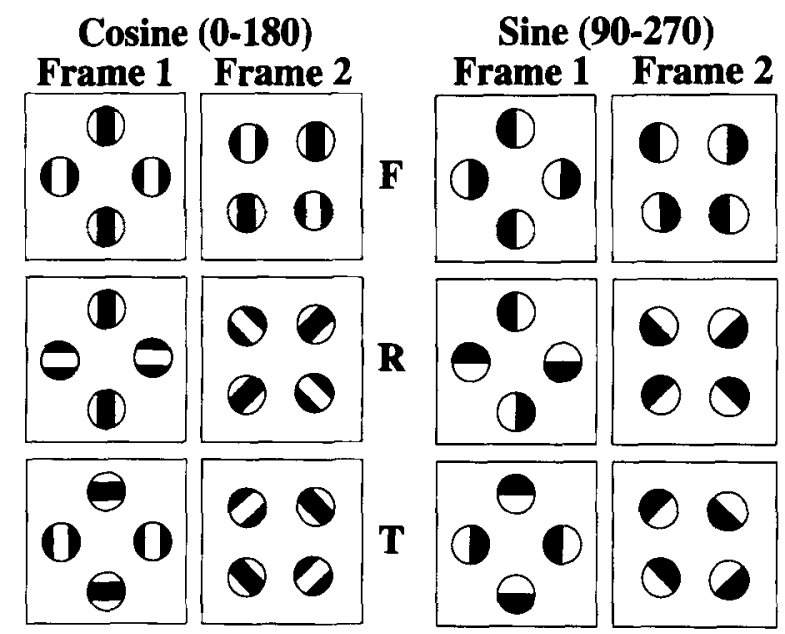

Figure 4. Schematic representations of two frames for each of the six conditions. Cosine phase conditions are represented in the two left columns, and sine phase in the two right columns. Ferris wheel (F), radial (R), and tangential ( $T$ ) conditions are represented in the top, middle, and bottom rows, respectively. Note that the experimental stimuli were actually Gabor patches, as in Figure 3.

proximately $1.8^{\circ}$. A black dot was always present in the center of the display to help the subjects maintain constant fixation.

Procedure. In each trial, eight frames of an apparent motion sequence were presented. In each frame, two pairs of $f+2 f$ Gabor patches were displayed in a circular array for $105 \mathrm{msec}$. During the ISI between frames, the screen was uniform in luminance, $L_{\text {avg }}$, except for the fixation dot. Figure 3 illustrates four frames of the eight-frame sequence for one cosine phase and one sine phase condition. From one frame to the next, the Gabor patches were repositioned $45^{\circ}$ clockwise or counterclockwise. Figures 3A-3D show clockwise movement for cosine phase stimuli, whereas Figures 3E-3H show counterclockwise movement for sine phase stimuli. The distance from the central fixation point to the center of each Gabor patch was $2.2^{\circ}$, and each Gabor patch moved $1.6^{\circ}$ (centerto-center distance) between frames. These parameters approximated those used in Green's (1986) experiment.

The subject's task was to report the perceived direction of motion: clockwise or counterclockwise. The "correct" direction was randomized from trial to trial. The subjects received no feedback about the "correctness" of their responses. If $180^{\circ}$ phase differences cannot be used to drive apparent motion, one would expect subjects to report clockwise and counterclockwise motion equally often. However, if subjects can use $180^{\circ}$ phase differences to drive apparent motion, one would expect subjects to report the "correct" direction of motion a significant proportion of the time. As previous researchers have pointed out (Mack et al., 1989; Pantle, Pinkus, \& Strout, 1992), the perceived direction of apparent motion on trial $n$ can be affected by the direction on trial $n-1$. We took two precautions to minimize any such hysteresis effects. First, we presented an extended sequence of apparent motion (eight frames), and the subjects were told to report only the very last direction they perceived. This gave the subject time to overcome directional biases lingering from the previous trial. Second, if the same "correct" direction were presented three times in a row, the next trial would always present motion in the opposite direction, and the response to that trial was not included in the analysis.

The two relative phase conditions, cosine and sine, were crossed with three different stimulus orientation conditions: Ferris wheel, radial, and tangential. These terms refer to the orientation of the compound grating stimuli with respect to the imaginary circle on which the patches were located. Figure 4 schematizes two frames for each of the six stimulus conditions. In the Ferris wheel conditions, gratings were always oriented vertically, regardless of patch location (top panels). In the radial conditions, the gratings were always oriented perpendicular to the tangent of the imaginary circle (middle panels). In the tangential condition, the gratings were always oriented parallel to the tangent of the imaginary circle (bottom paneis). So, in both radial and tangential conditions, each patch's orientation was rotated $45^{\circ}$ as it changed position from frame to frame.

We examined practice effects in 2 naive subjects (S.K. and S.M.), who completed $30-40$ blocks of 50 trials for each of the six conditions with one ISI: $45 \mathrm{msec}$ for S.K., and $75 \mathrm{msec}$ for S.M. S.K. then participated in the complete experiment along with three other observers (A.B.S., A.P., and R.W.). All were experienced subjects for this task, and S.K. and R.W. were naive with respect to the purpose of the experiment. These subjects completed eight blocks of 50 trials for each condition, with ISIs of $15,30,45,60$, 75,90 , and $105 \mathrm{msec}$.

\section{Results and Discussion}

Practice effects. Figure 5 shows the results of Ferris wheel conditions for Subject S.M. as a function of block number. Initially, performance was at chance for both cosine phase and sine phase conditions. Over the course of
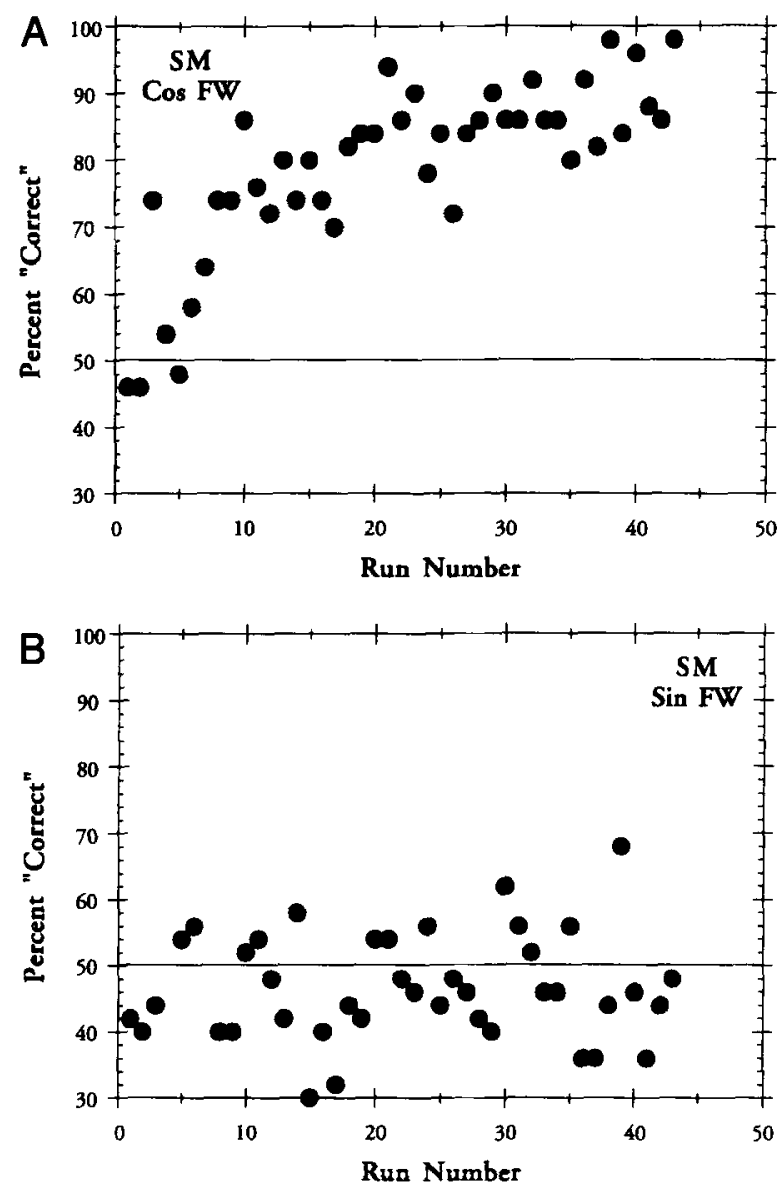

Figure 5. Results of practice for S.M. in the (A) cosine phase and (B) sine phase Ferris wheel conditions. Each point is based on 50 trials, and the horizontal bar represents chance performance. 
about 2,000 trials, performance on the cosine phase task improved dramatically, but performance on the sine phase task remained at chance. In other words, practice had a large effect on performance for the cosine phase task, but not for the sine phase task. This was true for both subjects, all stimulus orientations, and both ISIs tested. After extensive practice, it appeared that the subjects could use the cosine phase difference to drive apparent motion. However, even after extensive practice, the subjects could not reliably use the sine phase difference to drive apparent motion.

On the basis of the subjects' comments, it appears that much of the initial improvement on the cosine phase task was simply due to learning to fixate a central point while attending to peripheral targets. However, additional research on the extent to which learning transfers to other peripheral viewing tasks is clearly required to determine the nature of the learning involved. Regardless of the processes underlying these practice effects, it is important to note that practice does make a difference in the cosine phase task. Failure to provide subjects with adequate practice could lead to the erroneous conclusion that no phase differences can drive apparent motion.

Effects of ISI and orientation. Figure 6 shows the results of 4 subjects for all conditions as a function of ISI. In general, performance on the sine phase task was at chance, whereas performance on the cosine phase task was nearly perfect. There were no clear systematic effects of orientation or ISI, even for ISIs as long as $105 \mathrm{msec}$. The one exception to this general pattern can be seen in A.B.S.'s results. Although the cosine phase results for this subject were similar to those for the other subjects, performance on the radial and tangential sine phase tasks was generally higher than that on the same tasks for the other subjects. The difference was likely due to A.B.S.'s use of a grouping strategy rather than a difference in low-level processing of sine phase differences. The possible implications of grouping strategies are discussed in the General Discussion.

Navon (1976) suggested that the visual system processes different types of information at different rates and that, as stimulus durations increase and the visual
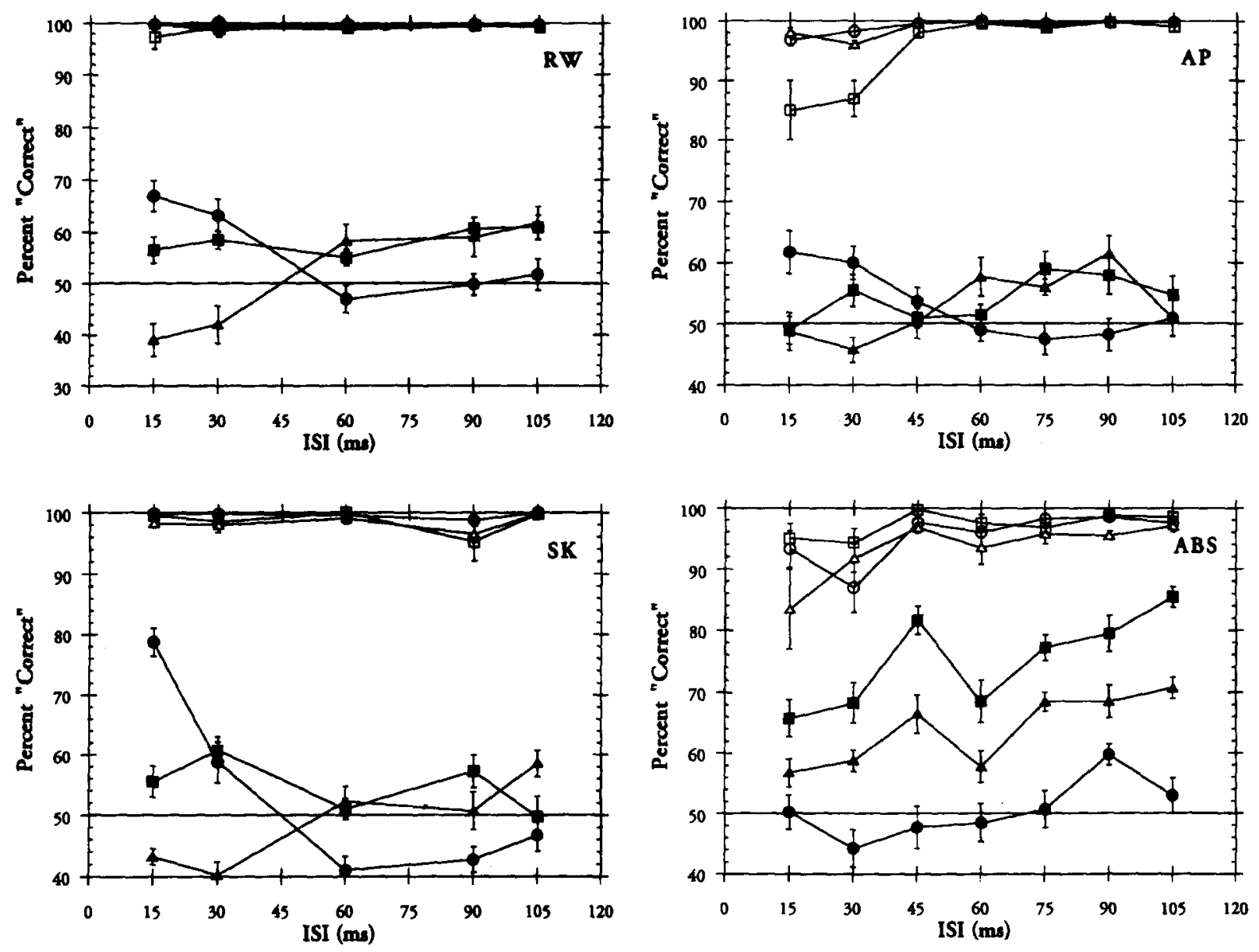

Figure 6. Percent "correct" for 4 subjects as a function of ISI (15-105 msec), phase shift (cosine or sine), and orientation (Ferris wheel, radial, or tangential). Open symbols represent results from cosine phase conditions; filled symbols represent results from sine phase conditions. Circles, square, and triangles represent results from the Ferris wheel, radial, and tangential conditions, respectively. Bars represent $\pm 1 S E$. Stimulus duration was constant at 105 msec. 
system is able to extract more information about stimuli, stimulus differences will be more likely to drive apparent motion. In terms of our stimuli, it was possible that sine phase differences were unable to drive apparent motion because sine phase differences were processed more slowly than were cosine phase differences. To test this hypothesis, 3 subjects (A.P., R.W., and S.K.) were tested in a subset of conditions with a 45-msec ISI and frame durations of 105, 210, and $315 \mathrm{msec}$. Figure 7 shows the results for the long-duration conditions. A comparison of Figures 6 and 7 indicates that the same general pattern of results holds even when frame duration is increased by a factor of 3 , so it is unlikely that the observers simply did not have enough time to extract form information from our sine phase stimuli.

The results from Experiment 1 suggest that cosine phase differences are effective in driving apparent motion, but sine phase differences are not. Furthermore, this same general pattern holds for all ISIs, durations, and orientations tested.

\section{EXPERIMENT 2}

In Experiment 1, we chose the range of frequencies to be optimal for our near-peripheral viewing conditions. However, because the range of spatial frequencies was relatively low, few cycles of the $f+2 f$ gratings were visible. Consequently, the stimulus bandwidth was relatively large. The goal of Experiment 2 was to determine the extent to which the ability of cosine phase differences to drive apparent motion could be generalized. Specifically, we wondered over what range of base frequencies and harmonic combinations cosine phase differences effectively drive apparent motion.

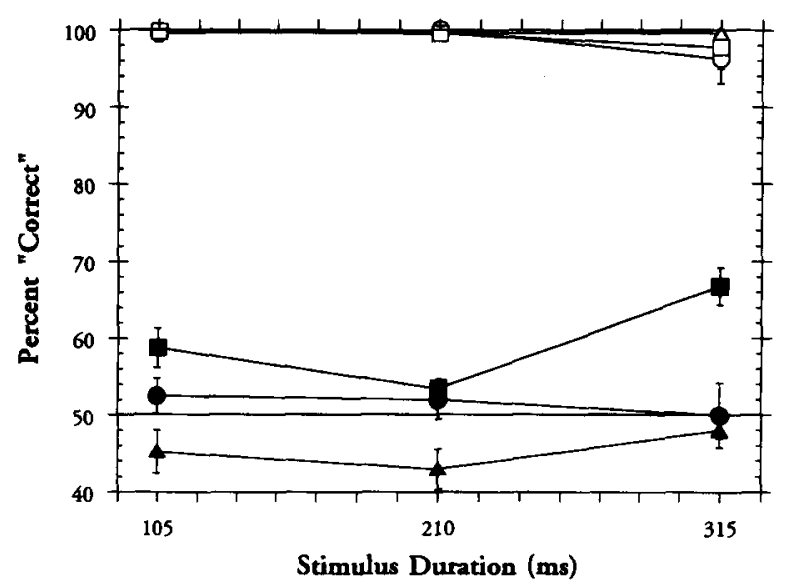

Figure 7. Percent correct for 3 subjects as a function of stimulus duration (105-315 msec). Open symbols represent results from cosine phase conditions; filled symbols represent results from sine phase conditions. Circles, triangles, and squares represent results for Subjects A.P., S.K., and R.W., respectively. Bars represent $\pm 1 S E$. ISI was constant at 45 msec.

\section{Method}

Subjects. Two subjects (A.B.S. and S.K.) participated in this experiment; the latter was naive with respect to all experimental hypotheses.

Stimuli and Procedure. The basic paradigm was identical to that of Experiment 1 with the following differences. Stimuli were Gaussian damped $f+n f$ compound sinewave gratings, where $n$ could be 2,3 , or 4 . Each of the three possible harmonic additions $(2 f, 3 f$, or $4 f)$ was crossed with three possible fundamental frequencies, $f(0.5,1.0$, or $1.5 \mathrm{cpd})$. In this way, the total spatial frequency range tested was from 0.5 to $6.0 \mathrm{cpd}$. The contrast of each spatial frequency was set to $30 \%$. In each of the nine possible cases, the harmonic, $n f$, was shifted either $0^{\circ}$ or $180^{\circ}$ relative to $f$ (cosine phase conditions), and the absolute phase was adjusted such that all stimuli were symmetric with respect to the center of the Gaussian envelope, as in Experiment 1. The frame duration was $105 \mathrm{msec}$, ISI was $45 \mathrm{msec}$, and the stimuli were oriented in the Ferris wheel configuration.

\section{Results and Discussion}

Figure 8 shows performance for the various fundamental frequencies as a function of harmonic combination. For the lowest fundamental frequency, $f=0.5 \mathrm{cpd}$, all harmonic combinations tested were effective in driving apparent motion. For higher base frequencies, $f=$ 1.0 or $1.5 \mathrm{cpd}$, the effectiveness of cosine phase differences in driving apparent motion decreased as the harmonic, $n$, increased. All $f+2 f$ cosine phase differences were able to drive apparent motion, but $f+3 f$ and $f+4 f$ cosine phase differences were most effective for the lowest fundamental frequency tested. So, for example, when $f$ was $1.5 \mathrm{cpd}$ and $3 f$ was added in $0^{\circ}$ or $180^{\circ}$ phase, the visual system could not use the cosine phase difference to drive apparent motion. Note that the cosine phase stimuli used by Green (1986) were similar to such a stimulus pair. Thus, although we have replicated Green's initial finding that this particular cosine phase difference is ineffective in driving apparent motion, we have shown that a range of other cosine phase differences can drive apparent motion.

The falloff in the effectiveness of $f+3 f$ and $f+4 f$ combinations to drive apparent motion as $f$ increases is not simply due to an increase in overall spatial frequency. One subject, A.B.S., was tested with $f+2 f$ compound gratings, where $f$ was $4.0 \mathrm{cpd}$. In this case, the highest component frequency was $8.0 \mathrm{cpd}$-higher than any of the frequencies tested in the main experiment. As the diamond in Figure 8A shows, performance was significantly greater than chance even though the range of spatial frequencies was relatively high for the eccentric viewing conditions.

The fact that with a fundamental frequency of $0.5 \mathrm{cpd}$ all harmonic combinations were able to drive apparent motion may simply be due to the broadband nature of the stimuli; this possibility is considered in the Results and Discussion section of Experiment 4. However, such an explanation cannot be used with respect to the finding that all $f+2 f$ combinations tested were able to drive apparent motion because $f$ varied from 0.5 to $4.0 \mathrm{cpd}$. We 

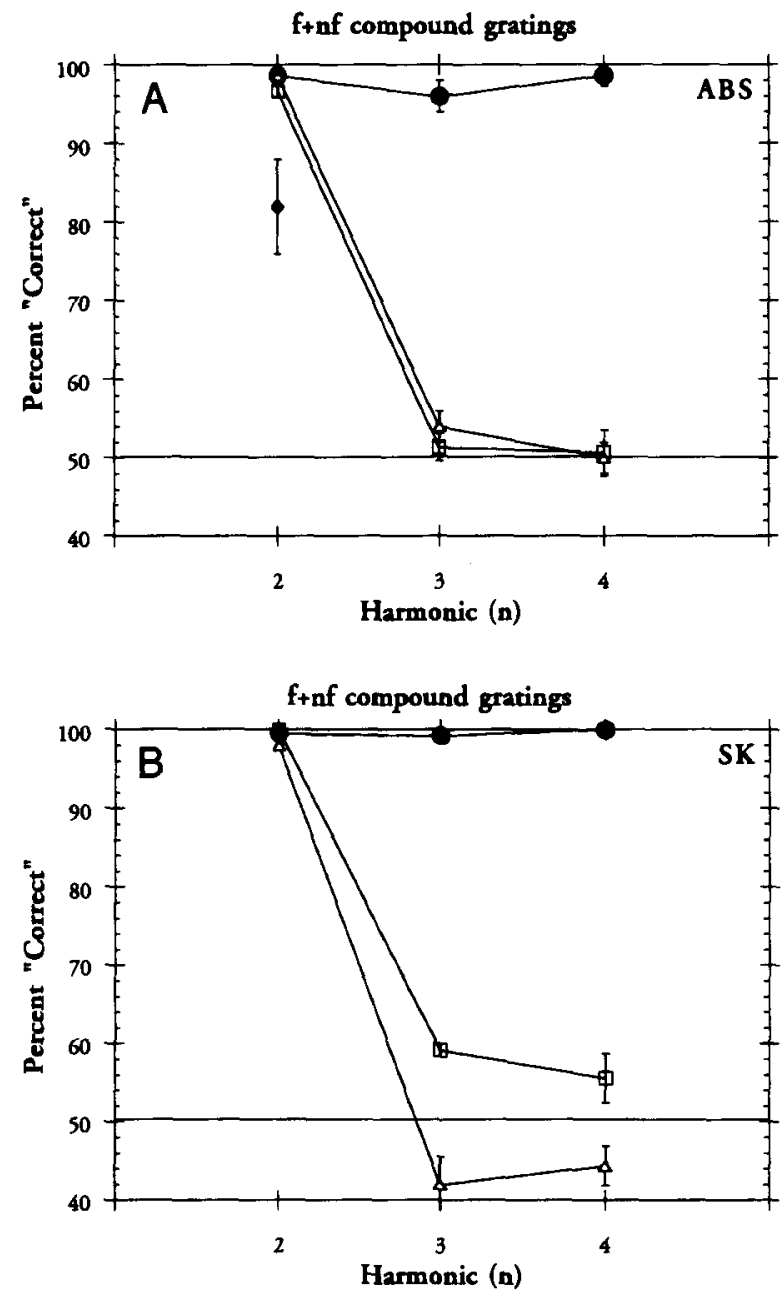

Figure 8. Percent correct for (A) A.B.S. and (B) S.K. as a function of base frequency $(f)$ and harmonic component $(n)$. Circles, squares, triangles, and diamonds represent results where $f$ equals $0.5,1.0,1.5$, and 4.0, respectively. Bars represent $\pm 1 S E$. Stimulus duration was 105 msec, and ISI was 45 msec.

suggest that the effectiveness of $f+2 f$ cosine phase differences and the relative ineffectiveness of $f+3 f$ and $f+4 f$ cosine phase differences is simply related to the perceptual similarity of stimuli. This hypothesis is explored in more detail in the General Discussion.

\section{EXPERIMENT 3}

Although the underlying goal of Experiments 1 and 2 was to determine the extent to which shape cues can be used to drive apparent motion, the cosine phase difference stimuli contain a cue in addition to shape differences: subjects sometimes report a small difference in perceived contrast between the different stimuli in the cosine phase pair. It was possible that the perceived contrast difference, rather than a shape difference per se, was driving apparent motion. In Experiment 3, we pitted contrast cues against shape cues to determine the relative

effectiveness of each in driving apparent motion. Specifically, the direction of motion based on contrast cues was opposite that based on phase or shape cues, and subjects were asked which direction dominated perception.

\section{Method}

Subjects. One author (A.B.S.) and 2 naive subjects (A.P. and S.K.) participated in the experiment.

Stimuli and Procedure. Figure 9 shows a schematization of two frames in the present experiment; note that the actual stimuli were Gaussian damped $f+2 f$ compound sinusoids, as before (see Figure 3). In Figure 9, the phase cue moves clockwise, while the contrast cue moves counterclockwise. As in Experiments 1 and 2, the subjects' task was to report the perceived direction of motion. Correct phase direction was randomized from trial to trial.

The frame duration was $105 \mathrm{msec}$, and ISI was $45 \mathrm{msec} . f+2 f$ cosine phase stimuli in the Ferris wheel orientation were tested, and $f$ was set to $0.5 \mathrm{cpd}$. In Figure 9, stimuli with greater contrast represent the standard stimuli, and stimuli of lesser contrast represent the comparison stimuli. The standard contrast levels of $f$ and $2 f$ were defined as $30 \%$, as in Experiments 1 and 2 . The comparison contrast of $f$ and $2 f$ could be set at six different levels: $30 \%$, $25 \%, 20 \%, 15 \%, 10 \%$, or $7.5 \%$. In each Gabor patch, $C_{f}$ was always set equal to $C_{2 f}$. The combination of standard and comparison contrast levels resulted in six standard:comparison ratios: 1.0, $1.2,1.5,2.0,3.0$, and 4.0. For a standard:comparison ratio of 1.0, one pair of stimuli was arbitrarily deemed the standard and the other the comparison.

\section{Results and Discussion}

Figure 10 shows the proportion of time that perceived direction is consistent with the phase cue, as a function of standard:comparison contrast ratios. Note that $50 \%$ on this graph indicates the equivalence point for contributions to apparent motion from phase and contrast cues. This equivalence point was estimated by probit analysis. All subjects reported seeing motion consistent with the phase cue until standard and comparison contrasts differed by about a factor of between 1.75 and 2.5 . Although there is some individual variation in the precise level at which the effectiveness of contrast and phase cues become equivalent, in all cases the phase cue dominates well beyond a small contrast difference. There-

FRAME 1

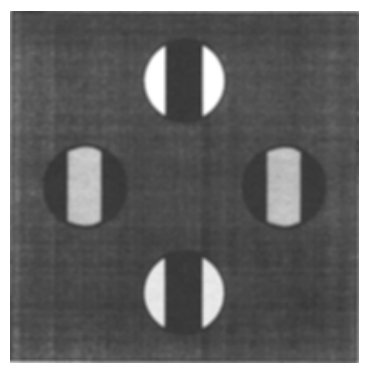

Figure 9. Schematic representation of two frames of the stimuli used in Experiment 3. For the stimuli shown here, the contrast cue moves counterclockwise, while the phase (shape) cue moves clockwise. The higher contrast stimuli are termed the standards, and lower contrast stimuli are termed the comparisons. Note that the stimuli were actually Gabor patches, as in Figure 3. 

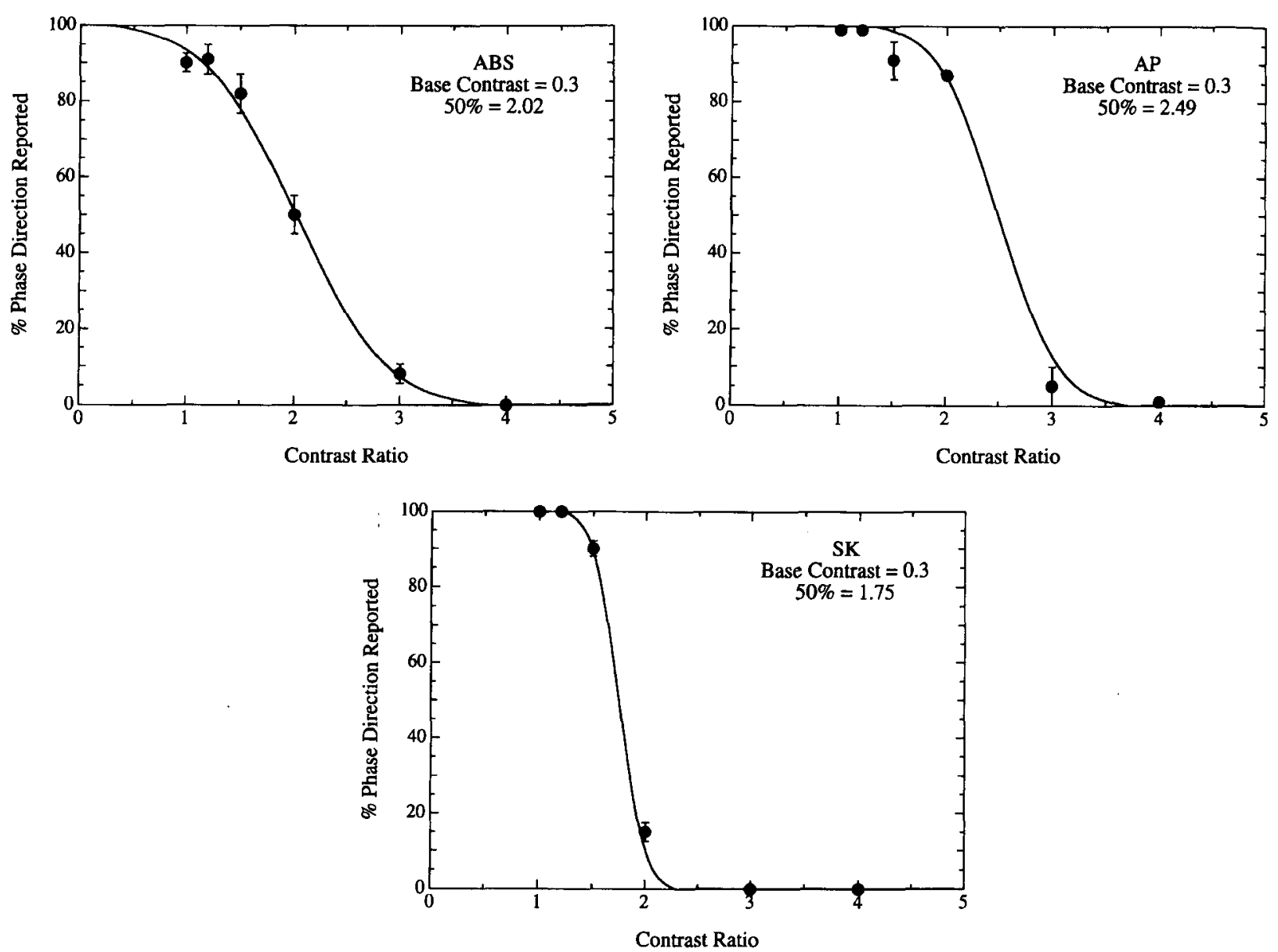

Figure 10. Percent correct phase direction as a function of contrast ratio. $50 \%$ indicates the contrast ratio for which subjects were equally likely to report motion based on the phase cue and motion based on the contrast cue. Bars represent $\pm 1 S E$. Stimulus duration was constant at $105 \mathrm{msec}$, and ISI was $45 \mathrm{msec}$.

fore, it is unlikely that the effectiveness of cosine phase differences in driving apparent motion is simply due to a perceived difference in contrast.

\section{EXPERIMENT 4}

In Experiments 1-3, stimulus contrasts were relatively high. All stimulus components in Experiments 1 and 2 and the standard stimulus component in Experiment 3 had contrasts of $30 \%$. It remains a possibility, then, that phase cues are useful in driving apparent motion only for very high contrast stimuli. The goal of Experiment 4 was to determine the extent to which the effectiveness of phase differences in driving apparent motion could be generalized over a range of contrast levels.

\section{Method}

Subjects. Subjects A.P. and S.K. participated in this experiment; S.K. was naive with respect to the experimental hypotheses.

Stimuli and Procedure. The stimuli were identical to those in Experiment 3, except that there were three standard contrast levels: $30 \%, 15 \%$, and $7.5 \%$. A standard:comparison ratio of 1.0 was tested for all standard contrast levels, a standard:comparison ratio of 2.0 was tested for standard contrast levels of $30 \%$ and $15 \%$, and a standard:comparison ratio of 4.0 was tested for a standard contrast level of $30 \%$.

\section{Results and Discussion}

As seen in Figure 11, the proportion of trials on which subjects reported motion to be consistent with the correct phase direction did not depend on the standard contrast level. It appears that phase differences can drive apparent motion over a wide range of contrasts. In addition, the results suggest that the equivalence point for phase and contrast cues may be invariant across different base contrast levels. However, additional testing is required with high standard:comparison ratios at low contrast levels to verify this suggestion.

The results of Experiment 4 suggest that phase differences are effective over a large range of stimulus contrasts. In addition, the results are useful in controlling for the potential confound of residual luminance artifacts. In our experiments, some of the phase differences that drove apparent motion comprised stimuli whose average lumi- 

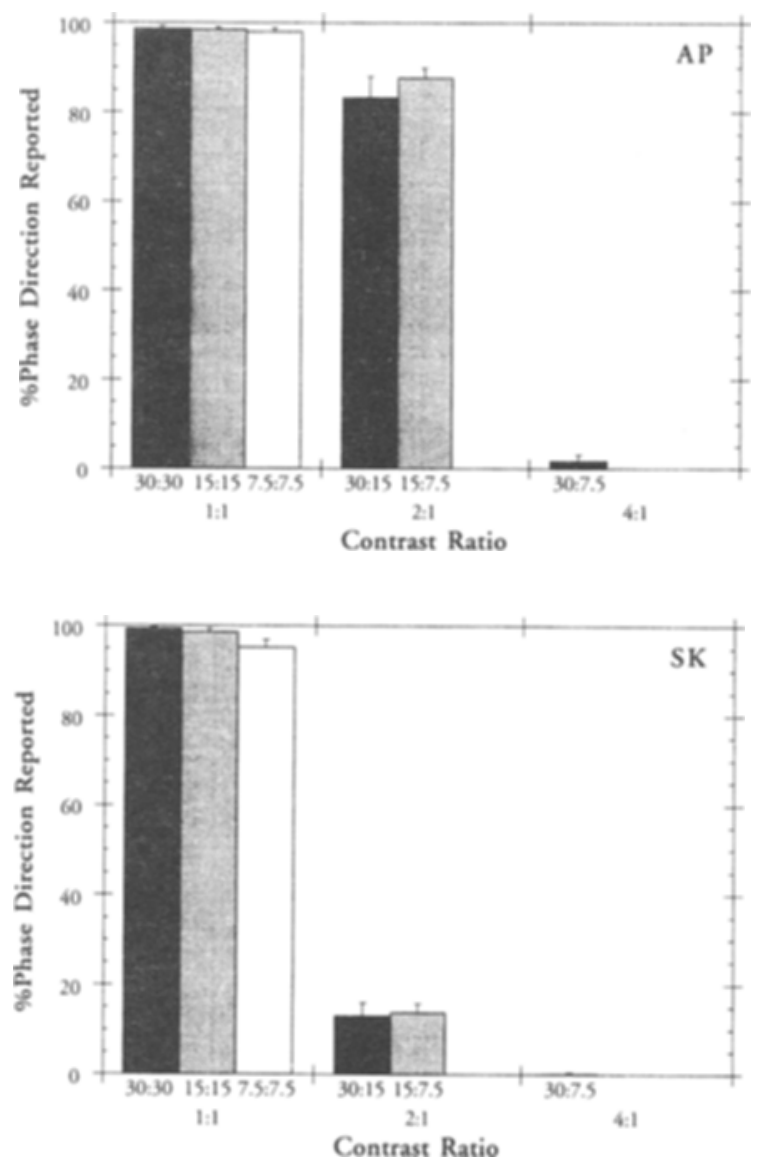

Figure 11. Percent correct phase direction as a function of contrast ratio and base contrast. Bars represent the $S E$. Stimulus duration was constant at $105 \mathrm{msec}$, and ISI was $45 \mathrm{msec}$.

nance differed slightly from that of the background, particularly when the stimuli contained relatively few cycles of the fundamental. In other words, some stimuli containing "dark bars" were slightly darker than the background, whereas some stimuli containing "light bars" were slightly lighter than the background. Because previous researchers have shown that luminance contrast and contrast polarity can be used to drive apparent motion (Anstis \& Mather, 1985; Green, 1989), we wanted to ensure that our effect was not entirely due to such an artifact of the stimuli.

To this end, we calculated residual luminances for all conditions in Experiments 1-4. Stimuli with higher fundamental frequencies (i.e., 1.0, 1.5, and $4.0 \mathrm{cpd}$ ) contained enough cycles to eliminate any luminance artifacts, with residual luminances of essentially $0 \%$. These results immediately suggest that not all of our results are due to residual luminance artifacts. However, the possibility remains that residual luminance artifacts were primarily responsible for the perception of apparent motion when the fundamental frequency was relatively low $(0.5 \mathrm{cpd})$ and the stimulus contained relatively few cycles. Table 1 shows the average luminances of these stimuli. If these small residual luminance artifacts were the driving force behind the perception of apparent motion, one would expect that as residual luminance decreased, so would the consistent perception of apparent motion. As seen in Table 1, the size of the residual luminance artifact decreases as stimulus contrast decreases. However, stimulus contrast does not affect the perception of apparent motion (see Figure 11). Clearly, then, residual luminance artifacts do not fully explain our results, even for stimuli with low fundamental frequencies.

\section{EXPERIMENT 5}

Previous researchers (Bennett, 1993; Bennett \& Banks, 1987, 1991; Field \& Nachmias, 1984; Kahana \& Bennett, 1994) have shown that phase discrimination for static gratings is well described by a two-channel model: one channel sensitive to cosine phase differences, and the other sensitive to sine phase differences. When the contrast of $f$ is held constant, one can determine the contrast of the harmonic (e.g., $2 f$ ) required to discriminate cosine $\left(0^{\circ}-180^{\circ}\right)$ and sine $\left(90^{\circ}-270^{\circ}\right)$ phase differences. Intermediate phase differences (e.g., $30^{\circ}-210^{\circ}$ ) are discriminable whenever the contrast of the harmonic exceeds either the cosine phase or the sine phase threshold. Within this framework, thresholds for phase discrimination can be plotted in the form of a box, as indicated in Figure 12. Here, the distance from each point to the origin, $c$, represents the contrast of the harmonic component needed for threshold phase discrimination, and the angle of each point from the abscissa, $\varnothing$, indicates the base phase of the harmonic component. The two-channel model simply predicts that all thresholds should lie along two lines described by a constant cosine phase amplitude (vertical line) and a constant sine phase amplitude (horizontal lines), as illustrated in the left panel of Figure 12. For certain viewing conditions and in certain individuals, the box is stretched vertically so that ratio of the sine:cosine thresholds increases, and, at some point, the sine phase task may become impossible altogether (Bennett, 1993; Bennett \& Banks, 1987, 1991), as illustrated in the right panel of Figure 12. There also appear to be large individual differences in discriminability and the rate at which the sine phase task becomes more difficult (e.g., compare Observers B.A. and A.B.S. in Figure 6 of Bennett \& Banks, 1991).

As a first step toward understanding the mechanisms underlying the ability of phase differences to drive ap-

Table 1

Residual Luminance Artifacts for Cosine Stimuli With $f=0.5 \mathrm{cpd}$

\begin{tabular}{|c|c|c|c|c|c|c|}
\hline \multirow[b]{3}{*}{ Base Phase } & \multicolumn{6}{|c|}{ Stimulus Contrast } \\
\hline & \multicolumn{2}{|c|}{$30 \%$} & \multicolumn{2}{|c|}{$15 \%$} & \multicolumn{2}{|c|}{$7.5 \%$} \\
\hline & $M$ & $\%$ & $M$ & $\%$ & $M$ & $\%$ \\
\hline 0 & 33.62 & +1.88 & 33.31 & +0.94 & 33.16 & +0.47 \\
\hline 180 & 32.38 & -1.88 & 32.69 & -0.94 & 32.84 & -0.47 \\
\hline
\end{tabular}

Note $-M$, mean luminance $\left(\mathrm{cd} / \mathrm{m}^{2}\right)$ of the stimulus. $\%$, percent luminance difference from the background. Background luminance was always $33 \mathrm{~cd} / \mathrm{m}^{2}$. 

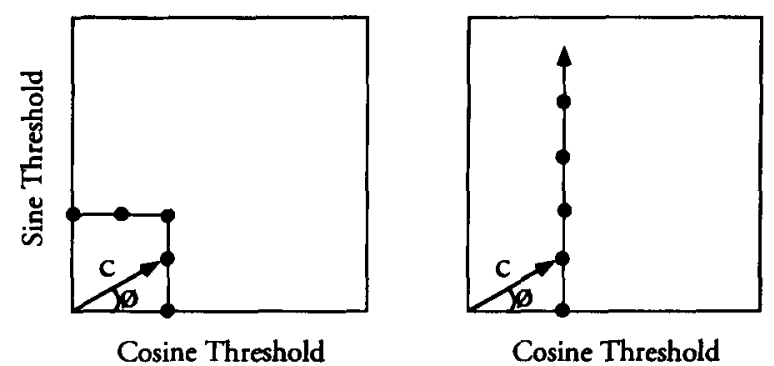

Figure 12. Schematic illustration of typical results from static phase discrimination experiments. The left panel represents a condition where observers are equally sensitive to cosine phase and sine phase discriminations (e.g., $f+2 f$ presented foveally). The right panel represents a condition where observers are relatively insensitive to sine phase discriminations (e.g, $f+2 f$ presented peripherally). See text for details. $c$, threshold contrast; $\phi$, base phase angle.

parent motion, Experiment 5 explored the plausibility of applying the two-channel model of static phase discrimination to the realm of apparent motion.

\section{Method}

Subjects. A.B.S. and A.P. participated in this experiment. A.P. was naive with respect to the experimental predictions.

Stimuli and Procedure. Frame duration was $105 \mathrm{msec}$, and ISI was $45 \mathrm{msec}$. As before, stimuli were Gaussian damped $f+2 f$ compound sinewave gratings in the Ferris wheel orientation. The fundamental frequency was set to $1.5 \mathrm{cpd}$, and $C_{f}$ was $30 \% . C_{2 f}$ was varied by the method of constant stimuli, and probit analysis was used to determine the $75 \%$ threshold - the contrast required for "correct" direction identification $75 \%$ of the time. Five $180^{\circ}$ phase differences were tested: $0^{\circ}-180^{\circ}, 15^{\circ}-195^{\circ}, 30^{\circ}-210^{\circ}, 45^{\circ}-225^{\circ}$, $60^{\circ}-240^{\circ}$.

\section{Results and Discussion}

In Figure 13, as in Figure 12, the distance from the origin to each symbol represents a $2 f$ threshold contrast, and the angle each symbol makes with respect to the abscissa represents the base phase for that $180^{\circ}$ phase dif- ference. The solid line represents a constant cosine phase amplitude. Providing the sine channel does not contribute to the perception of apparent motion, the two-channel model predicts that all thresholds should fall along a vertical line in the plots.

For both observers, "correct" direction thresholds were well described by a constant cosine phase amplitude (solid line). Thus, it appears that the two-channel model based on the results of static discrimination experiments may be a useful tool in the realm of motion perception. These results suggest that the motion system receives information from the same mechanisms that are responsible for static phase perception. However, additional research is clearly required to verify such a claim and to determine whether the mechanisms responsible for our perception of sine phase differences can ever feed into the apparent motion system.

Finally, it is interesting to note that when the contrast of $2 f$ was relatively low or null, observers could not reliably judge the "correct" direction of motion. This result essentially replicates Green's (1986) finding that, when there are multiple cycles in the stimulus, observers are unable to use absolute phase as a cue for solving the correspondence problem. Such a finding is important because it implies that the apparent motion does not rely on simply matching local luminance values for comparable positions across stimuli.

\section{EXPERIMENT 6}

Although we directly manipulated the relative phase of stimuli in Experiments 1-5, that manipulation was correlated with changes to the stimuli in addition to changes in perceived stimulus shape (see Badcock, 1984a, 1984b; Bennett, 1993). Experiments 2-4 ruled out any significant effect of contrast or average luminance artifacts, and the results of Experiment 5 suggest that apparent motion is not determined by simply matching
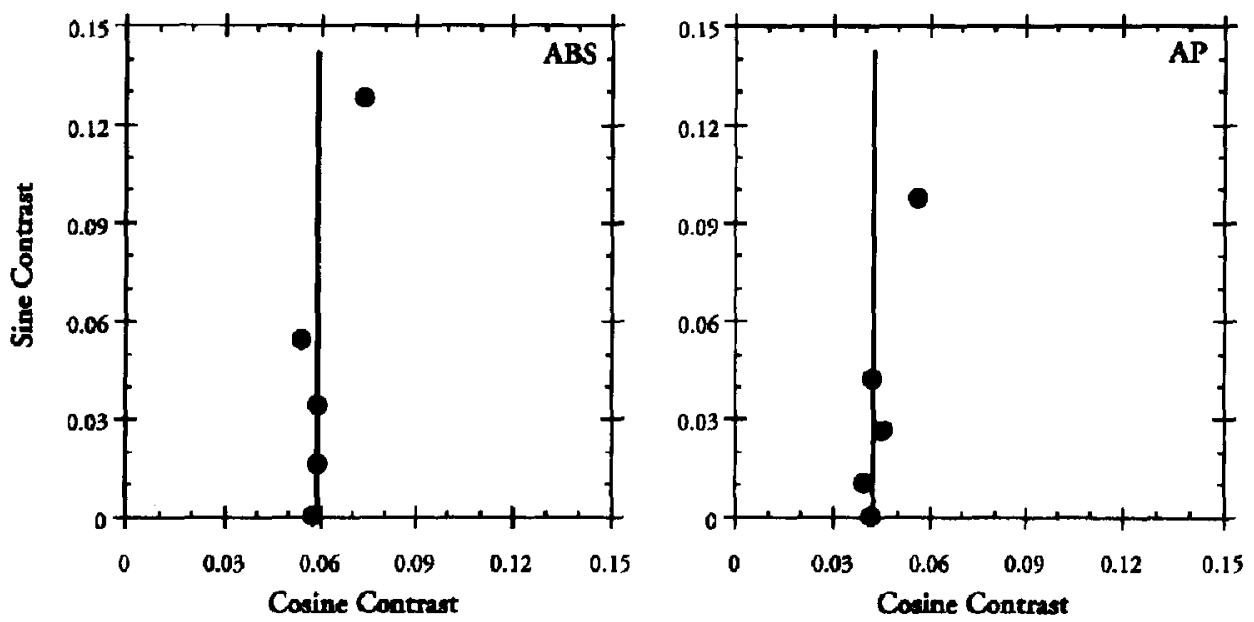

Figure 13. "Correct" direction thresholds for $2 f$ contrast in $f+2 f$ compound gratings. Cosine phase and sine phase contrasts are represented on the abscissa and ordinate, respectively. The vertical lines indicate a constant cosine phase contrast and represent the predictions of the two-channel model for static phase discrimination. $f=1.5 \mathrm{cpd} ; c_{f}=3$. 
local luminance values at comparable positions across stimuli. However, the effects of luminance maxima and minima still have not been addressed. For sine stimuli, when no consistent apparent motion was perceived, all stimuli contained the same maximum and minimum luminances. For cosine stimuli, when consistent apparent motion was perceived, maximum luminances were always greatest for stimuli in which $\emptyset_{r}=0^{\circ}$, and minimum luminances were always least for stimuli in which $\emptyset_{r}=$ $180^{\circ}$. The perception of apparent motion, therefore, could be explained by matching stimuli with similar maximum or minimum luminances.

Experiment 6 used stimuli with identical maximum, minimum, and average luminances. If apparent motion is determined by matching luminance maxima or minima, then we should find no consistent percept of apparent motion for these stimuli. If, on the other hand, phase (shape) differences drive apparent motion, then perceptually similar stimuli should be perceived to move to one another consistently.

\section{Method}

Subjects. Two subjects (A.B.S. and F.C.) participated in this experiment. F.C. was naive with respect to the experimental hypotheses and had not participated in any of the previous experiments.

Stimuli and Procedure. The basic paradigm was identical to that of Experiment 1 with the following exceptions. Each stimulus contained a square wave grating viewed through a $1.6^{\circ} \times 1.6^{\circ}$ square aperture. The stimulus was positioned within the aperture such that either the center of the bright bar or the center of the dark bar coincided with the aperture's center. The former condition is referred to as the positive square wave condition, and the latter is referred to as the negative square wave condition. The aperture was wide enough to show just one cycle of the grating, so the average luminance was identical for both patterns. For both types of stimuli, the maximum luminance was $43 \mathrm{~cd} / \mathrm{m}^{2}$ and the minimum was $23 \mathrm{~cd} / \mathrm{m}^{2}$, yeilding an average luminance of $33 \mathrm{~cd} / \mathrm{m}^{2}$ and a contrast of $30 \%$.

The observers viewed the stimuli binocularly from a distance of $82 \mathrm{~cm}$, with a head/chinrest stabilizing the head. The distance from the center of the display to the center of each patch subtended $2.2^{\circ}$. Stimulus duration was either 105,210 , or $315 \mathrm{msec}$, and ISI was always $45 \mathrm{msec}$. Stimuli were oriented in the Ferris wheel configuration. A.B.S. completed six block-randomized sets of each stimulus duration, and F.C. completed eight block-randomized sets.

\section{Results and Discussion}

Figure 14 shows the performance of each observer as a function of both block number and stimulus duration. As seen in Figure 14, both observers were able to reliably use stimulus differences to drive apparent motion. F.C., who was an unpracticed observer, developed this ability over the course of several blocks, as would be predicted from the results of Experiment 1 (see Figure 5A). Considering only the final four blocks for each observer, A.B.S. perceived the "correct" direction $83.7 \%$ of the time $(S E=2.6)$, and F.C. perceived the "correct" direction $95.0 \%$ of the time $(S E=1.7)$. There was no consistent effect of stimulus duration.

Because the stimuli were developed such that both positive and negative square waves contained the same luminances, the observers cannot have solved the correspondence problem reliably by matching stimuli with the same maximum or minimum luminances. If matches of luminance minima and maxima were solely responsible for driving apparent motion, we would have expected both observers to perceive the "correct" direction only $50 \%$ of the time throughout the duration of the experiment. Instead, both observers were able to perform the task with a high degree of consistency. This result shows that the perception of apparent motion is not determined by solely matching luminance maxima or minima. Instead, the results are consistent with the notion that phase (shape) differences drive apparent motion. It is not clear what aspects of shape differences were responsible for our results. One possibility is the absolute phase difference between positive and negative square wave stimuli was used to drive apparent motion. Such an interpretation seems to contradict the results of Experiment 5, which suggested that absolute phase difference cannot be used to drive apparent motion for stimuli containing multiple cycles. However, the present results may simply indicate that absolute phase difference can drive apparent motion under limited conditions (e.g., when only one
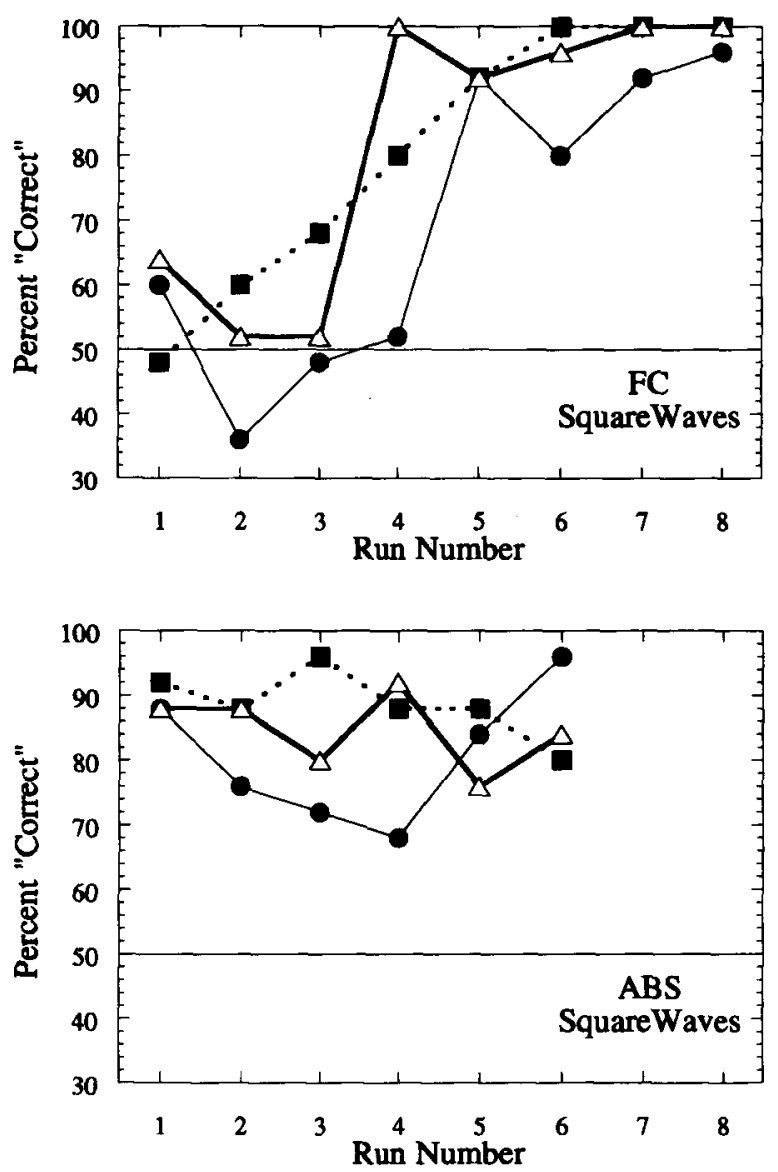

Figure 14. Percent correct perception of apparent motion for square wave stimuli as a function of run number. Each point is based on the result of 50 trials. Circles, squares, and triangles represent results for stimulus durations of 105,210 , and 315 msec, respectively. 
cycle of a stimulus is present). Alternatively, the observers' percepts may have been based on some other featural difference, such as the widths of black and white bars. The determinants of apparent motion are discussed further in the General Discussion.

\section{GENERAL DISCUSSION}

The claim by previous researchers that shape differences do not play a significant role in driving apparent motion was based primarily on the results of experiments using complex and variable stimuli. Our results, like those of some previous researchers (e.g., He \& Nakayama, 1994; Mack et al., 1989; Shechter et al., 1988), suggest that element appearances can play a significant role in the assignment of motion correspondence matches when proximity cues are not available. This does not mean that apparent motion can never be seen between two different shapes: Obviously, under many circumstances, it can (see Kolers \& Pomerantz, 1971). Additionally, when alternate cues (proximity, orientation, color, motion energy, etc.) are pitted against shape differences, the alternate cue can dominate (e.g., Nishida, Ohtani, \& Ejima, 1992; Nishida \& Takeuchi, 1990; Shechter et al., 1988; Werkhoven et al., 1993; Werkhoven, Snippe, \& Koenderink, 1990). Note, however, that the dominance of one cue over another in such conflicting situations does not imply that the motion system is insensitive to the dominated cue. To understand the mechanisms responsible for the perception of apparent motion, one needs to understand the interactions among and relative effectiveness of various cues, including shape, proximity, orientation, and so on.

Dawson (1991) described a model that began to address the issue of interaction among various cues. However, Dawson contended that "element appearances play, at best, a minor role in the assignment of motion correspondence matches and therefore should not represent a major component of a motion correspondence model" (p. 571). Instead, Dawson's model solves the correspondence problem based on three constraining principles: (1) element displacement should be minimized, (2) relative velocity should be minimized, and (3) element integrity should be maintained. Dawson suggested that shape differences could be incorporated into his model if their input were negatively weighted by the degree of figural change. While our results support the notion that element identity should be incorporated into models such as Dawson's, how one determines the "degree of figural change" has been unclear.
At first glance, it seems as though degree of figural change may be related to the discriminability of static phase differences. Table 2 summarizes results from the various conditions tested in the present study, along with results from static discrimination experiments from Bennett (1993) and Bennett and Banks (1991). Note that whenever static targets are easily discriminable they can drive apparent motion. Stimuli that are difficult or impossible to discriminate as static targets, such as those used by Green (1986), do not drive apparent motion.

As a first step in exploring the relationship between stimulus discriminability and apparent motion, we measured discrimination thresholds in one observer (A.B.S.). The target was a frame from the Ferris wheel orientation condition in Experiment 1. In the cosine phase condition, the target could be Figure 3B or Figure 3D; in the sine phase condition, the target could be Figure $3 \mathrm{~F}$ or $3 \mathrm{H} .{ }^{5}$ The target was presented for $105 \mathrm{msec}$, followed by a 45-msec ISI and a masking stimulus, after which the observer indicated which of the two targets had been presented. For each phase condition, cosine and sine, the mask on any trial was randomly chosen from two possible masking stimuli. A masking stimulus comprised four identical Gabor patches, each of which was one of the compound gratings from the previously presented target. Performance on the eight-frame apparent motion task was also remeasured with comparable stimulus durations and ISIs (105 and $45 \mathrm{msec}$, respectively). For both the apparent motion and the discrimination tasks, cosine phase and sine phase conditions were block randomized.

Figure 15 shows performance on discrimination and apparent motion tasks. for both phase conditions. If discriminability per se were the critical factor in determining the extent to which stimulus differences can drive apparent motion, then we would expect performance on apparent motion and discrimination tasks to be correlated with one another. As Figure 15 shows, this is not always the case. Performance on both discrimination and apparent motion tasks was above $90 \%$ for cosine phase targets. However, although performance on the apparent motion task was at chance for the sine phase stimuli, as in our previous experiments, the discriminability of sine phase targets was not significantly lower than the discriminability of cosine phase targets. Although these are clearly preliminary results, the outcome of this experiment makes it unlikely that discrimination per se determines whether stimulus differences will drive apparent motion. It remains a possibility that something akin to

Table 2

Summary of Results From the Present Study and From Bennett (1993) and Bennett and Banks (1991)

\begin{tabular}{cll}
\hline Peripheral Targets & \multicolumn{1}{c}{$\begin{array}{c}\text { Static Targets } \\
\text { Discriminable? }\end{array}$} & \multicolumn{1}{c}{$\begin{array}{c}\text { Can Drive } \\
\text { Apparent Motion? }\end{array}$} \\
\hline$f+2 f$ cosine & Yes, easily & Yes \\
$f+3 f$ cosine & No, except for low fundamentals & No, except for low fundamentals \\
$f+4 f$ cosine & No, except for low fundamentals & No, except for low fundamentals \\
Sine & No, or only with difficulty & No \\
\hline
\end{tabular}


the ease of discrimination, such as perceived similarity, determines the degree of figural change (Orlansky, 1940).

A recent study by Kahana and Bennett (1994) is consistent with the notion that perceptual similarity may be the critical factor for our apparent motion results. Kahana and Bennett studied classification of compound sinusoids, and they showed that stimuli with sine phase differences are perceptually more similar than stimuli with cosine phase differences, even if all stimuli are above the discrimination threshold. Previous researchers have also suggested that perceptual similarity determines the extent to which two stimuli will be preattentively discriminable (Duncan \& Humphreys, 1989). So, if perceptual similarity is the key to whether shape differences can drive apparent motion, one would expect to find overlap between stimulus pairs that are preattentively discriminable and stimulus pairs that drive apparent motion. In fact, recent work on visual search and apparent motion for complex objects supports this notion (Enns \& Rensink, 1990, 1991; Khurana, 1992; Kleffner \& Ramachandran, 1992). Future research is needed to determine more precisely the relationship between the discriminability and similarity of elements and their effectiveness in driving apparent motion.

It is also important to determine the extent to which the present results can be generalized to more complex stimuli such as those traditionally used in the study of shape and motion-specifically, geometric forms and letters. If the multiple-channel model is to be useful, one must first determine the relative importance of different aspects of form (spatial frequency, phase, orientation, contrast) under conditions where discriminability is controlled. The ultimate goal is to predict which form differences will drive apparent motion based simply on information from the phase and amplitude spectra.

\section{Energy Versus Similarity}

Recently, Werkhoven et al. (1993) conducted a series of experiments in which two potential cues for solving

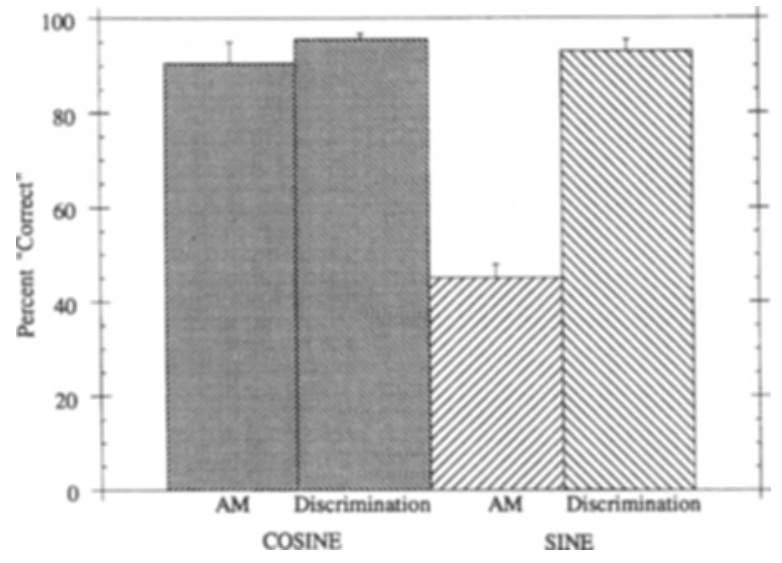

Figure 15. Percent correct for cosine phase differences and sine phase differences in apparent motion and discrimination tasks. Bars represent the $S E$. Stimulus duration was $105 \mathrm{msec}$, and ISI was 45 msec. the correspondence problem were pitted against one another: motion energy and stimulus similarity. Werkhoven et al. found that when these two cues conflicted, the motion percept seemed to be dominated by motion energy. The results of Nishida and Takeuchi (1990) and of Ramachandran et al. (1983) are also consistent with such a finding. On the basis of their results, Werkhoven et al. concluded that pattern similarity plays no role in apparent motion; instead, they suggested, the output of a single motion energy channel drives apparent motion. Werkhoven et al. (1993) state, "The visual motion system does not seem to be designed to establish correspondence between similar features in a motion sequence" (p. 481). However, although the results of Werkhoven et al. and others suggest that stimulus similarity may not be necessary to drive apparent motion, these results do not tell us whether stimulus similarity is sufficient to drive apparent motion. Werkhoven et al. find that when motion energy and stimulus similarity conflict, motion energy dominates our perception of apparent motion. However, this result does not logically lead to the conclusion that stimulus similarity never plays a role in the perception of apparent motion. The possibility remains that when motion energy and stimulus similarity yield the same percept, the two cues may work together synergistically. Furthermore, when motion energy is not available, the visual system may rely entirely on stimulus similarity to derive its percept.

The present experiments tested this latter hypothesis. Our stimuli prohibit reliable outputs from standard motion energy channels. This is because models for deriving motion energy ignore the relative phases of stimuli's component spatial frequencies (Adelson \& Bergen, 1985; van Santen \& Sperling, 1985; Werkhoven et al., 1993), and our stimuli differ only in the relative phases of their component spatial frequencies. In our experiments, the motion energy signal should be equal for all stimuli, regardless of perceived similarity. Therefore, on the basis of Werkhoven et al.'s claims, one would expect chance performance for all phase differences. In contrast, we found that cosine phase differences can drive apparent motion. Thus, our results imply that when the visual system cannot rely on the output of a motion energy channel, stimulus similarity (relative spatial phase) is, in fact, taken into account. The visual system can establish correspondence between similar features in a motion sequence.

Our results do not conflict with the results of Werkhoven et al. However, our conclusions are very different. Werkhoven et al. showed that differences in motion energy are sufficient to drive apparent motion. Our results show that stimulus similarity is sufficient to drive apparent motion. We suggest that the visual system contains at least two classes of mechanisms to solve the correspondence problem: one that relies on differences in motion energy, and another that relies on differences in stimulus similarity. It remains to be seen whether one mechanism always dominates or whether the two mechanisms work together when both cues would yield the same percept (as is the case with most real-world stimuli). 
The results of our experiments are not too surprising when considered in the context of recent discussions of first- and second-order motion (e.g., Boulton \& Baker, 1993a, 1993b; Cavanagh \& Mather, 1989; Chubb \& Sperling, 1988). It has become increasingly clear that observers can perceive apparent motion even when firstorder (Fourier) motion energy detectors do not yield a consistent response; this form of motion is termed secondorder. Because, as mentioned earlier, motion energy is determined without regard for relative phase, the motion perceived in the present experiments cannot be mediated by first-order motion mechanisms. Instead, second-order motion mechanisms must be responsible for the apparent motion percepts we found. As such, one might gain additional insights into the interactions between first- and second-order motion by covarying the amplitude and phase information contained in apparent motion displays.

\section{Determinants of Stimulus Similarity}

Although it seems clear that second-order motion mechanisms underlie our ability to use phase differences to drive apparent motion, the nature of those secondorder mechanisms remains unclear. As we have already noted, although we directly manipulated the relative phases of our component gratings, many other features of the stimulus were varied coincidentally (e.g., Badcock, 1984a). Bennett (1993) has argued that such featural changes play little, if any, role in phase discrimination for threshold tasks. However, local featural differences may be important in determining the relative similarity of our highly visible stimuli. Because relative phase shifts are intrinsically intertwined with many local featural changes, it is difficult to rule out the use of every possible cue. Nevertheless, our experiments can rule out the sole use of some featural cues for solving the correspondence problem. For example, Experiments 3 and 4 showed that a predicted percept of apparent motion can survive variations in the peak-to-trough amplitude of stimuli: Stimuli appeared to move to the position of the stimuli matched in relative phases, even when those same pairs visibly differed in overall amplitude. Experiment 5 showed that absolute phase shifts do not drive apparent motion, suggesting that the visual system does not solve the correspondence problem by matching local luminance values at comparable positions across stimuli. Finally, Experiment 6 showed that apparent motion can be reliably determined without matching luminance maxima or minima across stimuli.

It remains to be seen whether a parsimonious account of all our results can be predicted by some other featural cue or some combination of cues.

\section{Effects of Grouping and Other Higher Level Processing}

In Experiment 1, A.B.S.'s results differed from those of all other subjects. For A.B.S., cosine phase differences were still more effective in driving apparent motion than were sine phase differences. However, performance was above chance for sine phase differences in the radial and tangential conditions. In these conditions, A.B.S.'s sine phase results were consistently higher than those of the other subjects. After the experiments were completed, all subjects were asked their impression about what cues they were using. A.B.S. reported using a grouping strategy that was different from the other observers. Light regions and dark regions were grouped across Gabor patches so that the stimuli looked like one or two coherent structures, rather than four independent patches. Figure 16 illustrates the type of grouping percept described by A.B.S. The perception of direction appeared to be based on the global percept of the four stimuli, rather than on local information.

Another subject, S.M., was tested in sine phase and cosine phase conditions in radial and tangential orientations, and he actively searched for a strategy to perceive consistent motion. He was not told specifically what the strategy was; he was told simply that it was possible to consistently perceive the motion. Presentation durations were 105,210 , and $315 \mathrm{msec}$, and ISI was always $75 \mathrm{msec}$. As expected, his performance in the cosine phase conditions rapidly rose above chance, whereas his initial performance in the sine phase conditions was near $50 \%$ correct. We tracked S.M.'s block-by-block performance across conditions and noted the block in which he reported discovering a successful strategy for the sine phase task. Here, in a single block, his performance jumped from $50 \%$ to $80 \%$. Within the next block of trials, his improved performance generalized to all durations and orientations. After testing, he described using a grouping strategy similar to that used by A.B.S. In a sense, grouping in the present task is similar to the grouping of Street
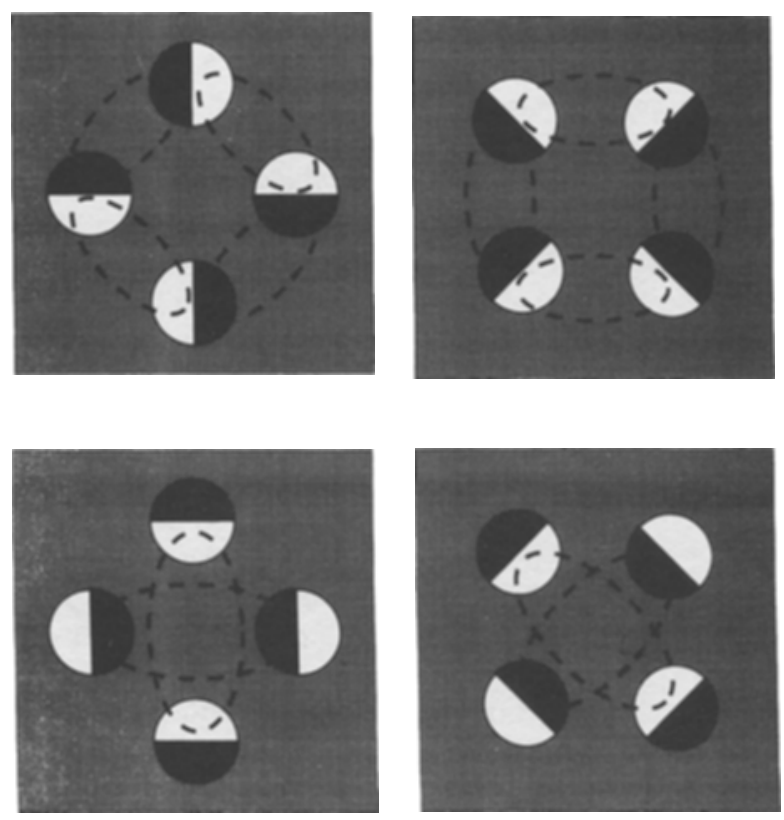

Figure 16. Schematic illustration of the phenomenal grouping strategy employed by Observers A.B.S. and S.M. Dashed ellipses represent grouped regions. Note that the stimuli were actually Gabor patches, as in Figure 3. 
figures (Street, 1931): It can occur spontaneously after familiarization with the stimuli; once the grouping is perceived, it is difficult to perceive the elements as individual objects again. Note, however, that the use of grouping strategies does not mean that sine phase stimuli per se can drive apparent motion. For example, grouping dark regions across elements, including the relatively bright region between these elements, may effectively excite low-spatial-frequency cosine phase detectors. We are currently exploring this hypothesis in more detail.

Another example of grouping in apparent motion comes from a study of shape from shading by Kleffner and Ramachandran (1992). They developed a variant of a random dot cinematogram in which each circular element was gradually shaded from top to bottom or from side to side. When the stimuli are brightest on top, they are perceived as "eggs"; when they are darkest on top, they are perceived as "holes." When the stimuli are brightest on the left or right, their depth interpretation is ambiguous. Note that all of these gradually shaded stimuli are essentially $180^{\circ}$ absolute phase differences. Previously, Green (1986) found that absolute phase differences cannot drive apparent motion, and we have verified his result informally (see Experiment 5). In Kleffner and Ramachandran's study, however, some absolute phase differences were effective in driving apparent motion. When several top-down shaded stimuli moved coherently among bottom-up shaded distractors, observers accurately perceived the direction of motion more than $85 \%$ of the time. Performance was significantly worse for side-shaded stimuli of opposite polarity. The authors argue that the visual system must assume that there is one lighting source located above the stimuli in order to achieve accurate apparent motion with top-to-bottom shaded stimuli. Once this assumption is made, stimuli can be grouped in terms of their depth percepts, and the motion of a distinct group of elements can be detected. In this case, our expectation about the source of lighting affects our ability to perceive coherent motion.

The use of grouping strategies in the perception of apparent motion raises the question of how higher level processes might affect perception. Our perception of motion, and of objects in general, clearly depends on the interaction between stimulus-derived information and a priori expectations (e.g., Ball \& Sekuler, 1981; Sekuler \& Sekuler, 1993; Sekuler, Sekuler, \& Sekuler, 1990). It seems likely that a priori expectations guide the extraction of stimulus-derived information, thus influencing our percepts. ${ }^{6}$ A dramatic example of this is seen in apparent motion when the stimuli are body parts (Shiffrar \& Freyd, 1991), rather than abstract forms. In this case, provided there is enough time between frames, the path of motion will not be the shortest but the most anatomically plausible. Previously acquired information about the structure and motion of limbs affects the perceived path of apparent motion. We are currently planning studies to determine more precisely the nature of the inter- actions between stimulus-derived and a priori information with respect to apparent motion and object perception in general.

\section{REFERENCES}

Adelson, E. H., \& Bergen, J. R. (1985). Spatio-temporal energy models for the perception of motion. Journal of the Optical Society of America A, 2, 284-299.

ANSTIS, S., \& MATHER, G. (1985). Effects of luminance and contrast on direction of ambiguous apparent motion. Perception, 14, 167-179.

BADCock, D. R. (1984a). How do we discriminate relative spatial phase? Vision Research, 24, 1847-1858.

BADCOCK, D. R. (1984b). Spatial phase or luminance profile discrimination? Vision Research, 24, 613-624.

Ball, K., \& Sekuler, R. (1981). Cues reduce direction uncertainty and enhance motion detection. Perception \& Psychophysics, 30, 1 19-128.

Banks, M. S., Sekuler, A. B., \& Anderson, S. J. (1991). Peripheral spatial vision: Limits imposed by optics, photoreceptors, and receptor pooling. Journal of the Optical Society of America A, 8, 17751787.

BennetT, P. J. (1993). The harmonic bandwidth of phase-reversal discrimination. Perception \& Psychophysics, 53, 292-304.

Bennett, P. J., \& Banks, M. S. (1987). Sensitivity loss among oddsymmetric mechanisms and phase anomalies in peripheral vision. Nature, 326, 873-876.

BenNETT, P. J., \& Banks, M. S. (1991). The effects of contrast, spatial scale, and orientation on foveal and peripheral phase discrimination. Vision Research, 31, 1759-1786.

Boulton, J. C., \& BaKer, C. L., JR. (1993a). Dependence on stimulus onset asynchrony in apparent motion: Evidence for two mechanisms. Vision Research, 33, 2013-2019.

BOULTON, J. C., \& BAKER, C. L., JR. (1993b). Different parameters control motion perception above and below a critical density. Vision Research, 33, 1803-1811.

Burt, P., \& Sperling, G. (1981). Time, distance and feature trade-offs in visual apparent motion. Psychological Review, 88, 171-195.

Caelli, T., Manning, M., \& Finlay, D. (1993). A general correspondence approach to apparent motion. Perception, 22, 185-192.

Carpenter, G. A., \& Grossberg, S. (1990). ART 3: Hierarchical search using chemical transmitters in self-organizing pattern recognition architectures. Neural Networks, 3, 129-152.

Carpenter, G. A., \& Grossberg, S. (1992). Fuzzy ARTMAP: Supervised learning, recognition, and prediction by a self-organizing neural network. IEEE Communications Magazine, 30, 38-49.

Cavanagh, P., \& Mather, G. (1989). Motion: The long and short of it. Spatial Vision, 4, 103-129.

Chubb, C., \& SperLing, G. (1988). Drift-balanced random stimuli: A general basis for studying non-Fourier motion perception. Journal of the Optical Society of America A, 5, 1986-2006.

DAwson, M. R. W. (1991). The how and why of what went where in apparent motion: Modeling solutions to the motion correspondence problem. Psychological Review, 98, 569-603.

DUNCAN, J., \& HUMPHREYS, G. W. (1989). Visual search and stimulus similarity. Psychological Review, 96, 433-458.

EnNs, J. T., \& RensinK, R. A. (1990, February 9). Scene-based properties influence visual search. Science, 247, 721-723.

EnNS, J. T., \& RENSINK, R. A. (1991). Preattentive recovery of threedimensional orientation in visual search. Psychological Review, 98 , 335-351.

Field, D. J., \& NaChmias, J. (1984). Phase reversal discrimination, $V i$ sion Research, 24, 333-340.

GrEeN, M. (1986). What determines correspondence strength in apparent motion? Vision Research, 26, 599-607.

Green, M. (1989). Color correspondence in apparent motion. Perception \& Psychophysics, 45, 15-20.

He, Z. J., \& Nakayama, K. (1994). Perceived surface shape not features determines correspondence strength in apparent motion. $\mathrm{Vi}$ sion Research, 34, 2125-2135.

KahANA, M. J., \& BENNETT, P. J. (1994). Classification and perceived 
similarity of compound gratings that differ in relative spatial phase. Perception \& Psychophysics, 55, 642-656.

KhuRANA, B. (1992). Scene-based versus image-based correspondence in apparent motion [Abstract]. Investigative Ophthalmology \& Visual Science, 33(Suppl.), 1143.

KlefFner, D. A., \& Ramachandran, V. S. (1992). On the perception of shape from shading. Perception \& Psychophysics, 52, 18-36.

Kolers, P. (1972). Aspects of motion perception. Oxford: Pergamon. Kolers, P., \& Pomerantz, J. (1971). Figural change in apparent motion. Journal of Experimental Psychology, 87, 99-108.

KoRIAT, A. (1994). Object-based apparent motion. Perception \& Psychophysics, 56, 392-404.

Mack, A., Klein, L., Hill, J., \& Palumbo, D. (1989). Apparent motion: Evidence of the influence of shape, slant, and size on the correspondence process. Perception \& Psychophysics, 46, 201-206.

Morrone, M. C., \& OWENS, R. (1987). Feature detection from local energy. Pattern Recognition Letters, 1, 103-113.

NAvoN, D. (1976). Irrelevance of figural identity for resolving ambiguities in apparent motion. Journal of Experimental Psychology: Human Perception \& Performance, 2, 130-138.

Nishida, S., OHTANI, Y., \& EJIMA, Y. (1992). Inhibitory interaction in a split/fusion apparent motion: Lack of spatial frequency selectivity. Vision Research, 32, 1523-1534.

NISHIDA, S., \& TAKEUCHI, T. (1990). The effects of luminance affinity of apparent motion. Vision Research, 30, 709-721.

ORLANSKY, J. (1940). The effect of similarity and difference in form on apparent visual motion. Archives of Psychology, 246, 1-85.

Pantle, A., Pinkus, A., \& Strout, J. (1992). Motion signal interactions [Abstract]. Investigative Ophthalmology \& Visual Science, 33(Suppl.), 973.

Piotrowski, L. N., \& CAMPbell, F. W. (1982). A demonstration of the visual importance and flexibility of spatial-frequency amplitude and phase. Perception, 11, 337-346.

Prazdny, K. (1986). What variables control (long-range) apparent motion? Perception, 15, 37-40.

RamaChandRan, V.S., GinsBurG, A., \& ANSTIS, S. M. (1983). Low spatial frequencies dominate apparent motion. Perception, 12, 457-461.

Rovamo, J., Virsu, V., \& NASANEN, R. (1978). Cortical magnification factor predicts photopic contrast sensitivity of peripheral vision. $\mathrm{Na}$ ture, 271, 54-56.

Sekuler, A. B., Persaud, A., \& Bennett, P. J. (1992). Do phase differences drive apparent motion? [Abstract] Investigative Ophthalmology \& Visual Science, 33(Suppl.), 1138.

Sekuler, A. B., \& SeKuler, R. (1993). Representational development of direction in motion perception: A fragile process. Perception, 22 , 899-915

Sekuler, A. B., Sekuler, R., \& Sekuler, E. B. (1990). How the vi- sual system detects changes in the direction of moving targets. Perception, 19, 181-195.

Shechter, S., Hochstein, S., \& Hillman, P. (1988). Shape similarity and distance disparity as apparent motion correspondence cues. $V i$ sion Research, 28, 1013-1021.

Shiffrar, M., \& Freyd, J. J. (1991). Apparent motion of the human body. Psychological Science, 1, 257-264.

StreEt, R. F. (1931). A gestalt completion test: A study of a cross section of intellect. New York: Bureau of Publication, Columbia University.

van Santen, J. P. H., \& Sperling, G. (1985). Elaborated Reichardt detectors. Journal of the Optical Society of America A, 2, 300-321.

Werkhoven, P., SNiPPE, H. P., \& KoEnderink, J. J. (1990). Effects of element orientation on apparent motion perception. Perception \& Psychophysics, 47, 509-525.

Werkhoven, P., SPERling, G., \& ChubB, C. (1993). The dimensionality of texture-defined motion: A single channel theory. Vision Research, 33, 463-485.

\section{NOTES}

1. A and B represent different stimulus patterns, not the actual letters $\mathrm{A}$ and $\mathrm{B}$.

2. Left and right edges refer to the direction of the steepest luminance gradient.

3. $0^{\circ}-180^{\circ}$ and $90^{\circ}-270^{\circ}$ refer to pairs of compound grating stimuli in which the relative spatial phase of the higher frequency component differs by $180^{\circ}$. The initial number in each term $\left(0^{\circ}\right.$ or $\left.90^{\circ}\right)$ is defined as the base phase. So, for example, a $0^{\circ}-180^{\circ}$ phase pair comprises two compound gratings: one in which the higher component is in $0^{\circ}$ phase relative to the lower component, and one in which the higher component is in $180^{\circ}$ phase relative to the lower component.

4. Let $F(x)=A 1^{*} \cos (r 1)+A 2^{*} \cos (r 2)$, where $r 1=2 \Pi f_{1} x-\emptyset a$ and $r 2=2 \Pi f_{2} x-\left(f_{2} / f_{1}\right) \emptyset_{a}-\emptyset_{r}$, and let $H(x)$ equal the Hilbert transform of $F(x)$. The pattern energy function is defined as $E(x)=F^{2}(x)+$ $H^{2}(x)$. For $f+2 f$ gratings, the energy function is periodic and has a peak wherever $r 1=r 2$.

5. To avoid potential confounds associated with radial and tangential grating presentation, stimulus configurations for targets were always those in which the patches were located on the obliques.

6 . This clearly is not a new idea, but see Carpenter and Grossberg, 1990,1992 , for relatively recent examples of a computational implementation of this type of interactive system.

(Manuscript received May 18, 1993; revision accepted for publication May 22, 1995.) 